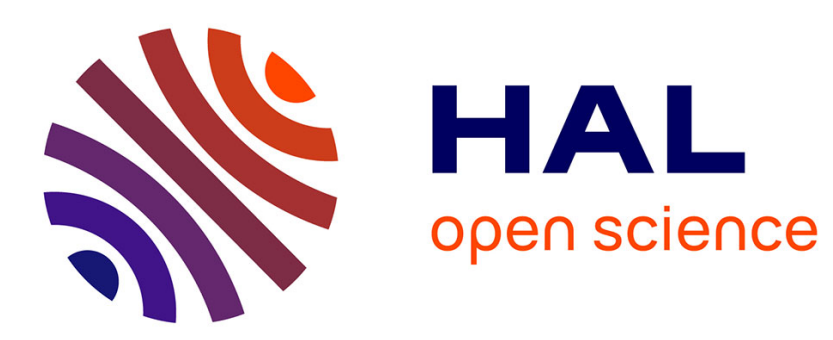

\title{
Taking into account thermal residual stresses in topology optimization of structures built by additive manufacturing
}

G. Allaire, L Jakabčin

\section{- To cite this version:}

G. Allaire, L Jakabčin. Taking into account thermal residual stresses in topology optimization of structures built by additive manufacturing. 2017. hal-01666081

\section{HAL Id: hal-01666081 \\ https://hal.science/hal-01666081}

Preprint submitted on 18 Dec 2017

HAL is a multi-disciplinary open access archive for the deposit and dissemination of scientific research documents, whether they are published or not. The documents may come from teaching and research institutions in France or abroad, or from public or private research centers.
L'archive ouverte pluridisciplinaire HAL, est destinée au dépôt et à la diffusion de documents scientifiques de niveau recherche, publiés ou non, émanant des établissements d'enseignement et de recherche français ou étrangers, des laboratoires publics ou privés. 


\title{
Taking into account thermal residual stresses in topology optimization of structures built by additive manufacturing
}

\author{
G. Allaire and L. Jakabčin \\ Centre de Mathématiques Appliquées, UMR CNRS 7641 \\ École Polytechnique, Université Paris-Saclay, 91128 Palaiseau, France. \\ Email: gregoire.allaire@polytechnique.fr, lukas.jakabcin@polytechnique.edu
}

December 12, 2017

\begin{abstract}
We introduce a model and several constraints for shape and topology optimization of structures, built by additive manufacturing techniques. The goal of these constraints is to take into account the thermal residual stresses or the thermal deformations, generated by processes like Selective Laser Melting, right from the beginning of the structural design optimization. In other words, the structure is optimized concurrently for its final use and for its behavior during the layer by layer production process. It is well known that metallic additive manufacturing generates very high temperatures and heat fluxes, which in turn yield thermal deformations that may prevent the coating of a new powder layer, or thermal residual stresses that may hinder the mechanical properties of the final design. Our proposed constraints are targeted to avoid these undesired effects. Shape derivatives are computed by an adjoint method and are incorporated into a level set numerical optimization algorithm. Several 2-d and 3-d numerical examples demonstrate the interest and effectiveness of our approach.
\end{abstract}

\section{Introduction}

Topology optimization methods have long suffered from a drawback, inherent to its main advantage: the computed optimal structures have so complex topologies that they are, very often, impossible to build by traditional techniques like casting. This has boosted many research works on the addition of manufacturing constraints during the optimization, see e.g. [4], [5], [17], [18], [19], [21], [37], [44]. The picture is completely different with the emergence and maturity of additive manufacturing technologies, which are able to build structures with a high degree of complexity, thereby allowing to process almost directly the designs predicted by shape and topology optimization algorithms [8], [16]. Although, in principle, additive manufacturing can build any kind of shapes, there remain some explicit or implicit constraints on their geometries and topologies. Let us quote a few of them: overhang limitations [2], [3], [11], [26], [27], alloy anisotropy [40], [45], orientation with respect to the build direction [28], no closed holes (in order to remove the powder inside), and finally thermal residual stresses or thermal deformations.

Here we focus on this last type of constraints which is typically associated with metallic powder bed additive manufacturing processes. Indeed, thermal residual stress is one of the main undesired effect that results from metallic additive manufacturing processes. They are generated by the large heat fluxes, and high temperatures, brought to the metallic powder by 
the laser or electron beam. They can yield large deformations of the built structure, when it is detached from its support, or even cause irreversible damage, fracture or delamination of the successive layers. Therefore, the prediction of thermal residual stress and its possible avoidance are key technological issues. There are already many works, either experimental or numerical, devoted to the study of thermal residual stress [15], [20], [23], [25], [30], [35], [36], [42], [43]. The numerical studies are based either on detailed models, which are very complex and computationally expensive since they are typically multi-physics and multi-scales, or on simplified models, which are relevant at a more macroscopic scale, thanks to some homogenization process. However, to the best of our knowledge, none of these models, even the simplest ones, have been yet incorporated into a shape and topology optimization loop. Our goal is thus to propose a model for the appearance of thermal residual stress and to suggest several objective functions or constraints to take them into account in shape and topology optimization of structures.

Since this model of thermal residual stress is going to be repeatedly called into an optimization loop, in order to avoid too expensive computational costs, we rely on a simplified modeling which, in particular, ignores details of phase change, annealing effects, any nonlinearities in heat exchange and mechanical irreversibility. Yet, it features some key issues: the presence of two phases, powder and solid, the layer by layer building process, time evolution of heat propagation following the beam path and thermo-mechanical response of the structure under construction. To this model are associated several possible constraints for the optimization. We focus on two different choices. A first constraint is an integral, in time and space, of the stress created during the building process, which should remain below some predefined threshold. A second constraint is linked to the vertical displacement of the upper boundary of each intermediate structures which must stay below a maximal gap distance so that the rake or roller (which coats a new powder layer on top of the previous intermediate structure) can advance without being stopped by the thermally deformed structure, acting as an obstacle.

The proposed model and constraints are related to the behavior of the structure during its building process. Of course, the final use of the structure has to be incorporated in the optimization too. Therefore, our shape and topology optimization problem features two state equations: one for the final use of the structure (typically, linearized elasticity and compliance as an objective function), another one for the sequence of intermediate structures during the building process (our thermal residual stress model). Of course, this has the consequence of making the optimization problem much more costly than in the absence of thermal residual stress constraints, which in turn explains our choice of a simplified model.

The content of the present paper is the following. In Section 2 our model of thermo-elastic evolution is described in details. To simplify the exposition, we first ignore the layer by layer building process. In other words, our model is described in the final configuration of the build chamber which is filled by two different materials or phases: powder and solid. Our model is made of two partial differential equations: the time dependent heat equation, coupled to the quasi-static thermoelastic equilibrium system. It is inspired by a model proposed in [42] (see also [30]), except that, for simplicity, plastic irreversible deformations are neglected and not taken into account (we plan to come back to the original model of [42] in a further work). An objective function for our simplified model depends on the position of the interface between the two phases, powder and solid. The corresponding adjoint problems are introduced which allow us to compute the shape derivative of this objective function (based on previous works [1], [7]). The main difficulties are twofold. First, it is an interface between two subdomains, and not the exterior boundary, which is optimized. Second, the adjoint problem for the heat equation is backward in time, which makes the numerical computation of the shape derivative quite expensive. Our main result is Theorem 2.4 which delivers the shape derivative of a generic objective function (in the simplified setting of a spatially discretized problem). Theorem 2.5 
gives a similar result in a discrete time setting.

Section 3 introduces the additional difficulty of the layer by layer building process. We follow the approach of [2], [3] to model the layer by layer construction. If the final solid structure is called $\Omega$, there are a sequence of intermediate structures $\Omega\left(t_{i}\right)$ corresponding to an increasing sequence of discrete times $t_{i}$, each corresponding to the addition of a new layer in the building process. Of course, in numerical practice several layers (which are very thin, typically of the order of 50 micrometers) can be lumped together in order to have a smaller number of intermediate structures to analyse. Each $\Omega\left(t_{i}\right)$ is simply the truncation of $\Omega$ at the height (in the building direction) attained at time $t_{i}$. Therefore, the only variable in the optimisation problem is the final structure $\Omega$, although the objective function and the constraints depend on state equations posed both in $\Omega$ and in $\Omega\left(t_{i}\right)$. Our main result is Theorem 3.1 which furnishes the shape derivative of the objective function for this multiple loads optimization problem. In a discrete time setting a variant of this result is Theorem 3.2 .

In Section 4 details are given about our numerical algorithm. The level set method is briefly recalled, as well as the regularization and extension process for the velocity in the HamiltonJacobi equation which is deduced from the shape derivative. The regularization of the interface between the solid and powder, proposed in [1], is also explained.

Finally, Section 5 is devoted to numerical results for a MBB beam in 2-d and 3-d. We consider two objective functions. The first one is the $L^{2}$-norm in space and time of the Von Mises stress (i.e. its deviatoric part) which is our proposed measure of thermal residual stresses in our simplified modeling. The second one is a measure of the vertical displacement above a given threshold in the upper layer of all intermediate shapes during the building process. We perform test cases for the simplified model of Section 2, as well as for the layer by layer model of Section 3. Since the computational time is quite large (compared to a standard compliance minimization problem), we always choose as an initialization a structure which has already been optimized for its final use, without taking into account the additive manufacturing process. We minimize one of the two above objective functions, keeping the volume fixed and with an inequality constraint on the compliance which must not increase too much. Our minimization algorithm works well in the sense that the objective functions are substantially reduced (say by almost $50 \%$ in many cases) while the constraints are satisfied at convergence. The optimized shapes are not very different from the initial ones (in particular, they share the same topology) and it is not intuitive to understand how the geometry changes influenced the decrease of the objective functions. Future work will be devoted to the exploration of other objective functions, trying other initializations (which may be very costly) and extending our layer by layer model to take into account plastic deformations.

\section{A thermo-elastic optimization problem}

The goal of this section is to introduce a simpler model than the complete one of the next Section 3 which, in particular, does not feature the layer by layer additive manufacturing process. However, the equations are the same. Therefore, this allows us to separate the difficulties and to simplify the exposition. This model is made of the coupling of the heat equation with a quasi-static thermo-elastic system. It is thus a simplified model since it ignores plastic deformations, as in [42], or the melt pool physics (including phase changes and annealing effects), as in [30]. This (possibly exaggerated) simplification is motivated by our requirement of limited computational cost in an optimization loop. Further, we present the various shape derivatives and required adjoint systems without taking into account the varying domain and material properties which will be introduced in Section 3 . 


\subsection{A thermo-elastic evolution model}

Throughout the paper, $\mathcal{D}$ is a bounded connected open set in $\mathbb{R}^{n}$ (typically, the build chamber) with Lipschitz boundary $\partial \mathcal{D}=\partial \mathcal{D}_{D} \cup \partial \mathcal{D}_{N} \cup \partial \mathcal{D}_{F}$ where $\partial \mathcal{D}_{D}, \partial \mathcal{D}_{N}, \partial \mathcal{D}_{F}$ are disjoint relatively open sets in $\partial \mathcal{D}$ with the (surface) measure of $\partial \mathcal{D}_{D}$ assumed to be positive. We denote $H_{D}^{1}(\mathcal{D}):=$ $\left\{u \in H^{1}(\mathcal{D}) ; u=0\right.$ on $\left.\partial \mathcal{D}_{D}\right\}$.

We suppose that $\overline{\mathcal{D}}=\overline{\Omega_{1}} \cup \overline{\Omega_{2}}$ where $\Omega_{1}$ and $\Omega_{2}$ are relatively open sets in $\mathcal{D}$ (typically, they correspond to the solid part and to the powder part). The interface between these two subsets is $\Gamma=\partial \Omega_{1} \cap \partial \Omega_{2}$ (see Figure 1). The subdomains $\Omega_{1}$ and $\Omega_{2}$ are filled by two different thermoelastic materials. All the mechanical parameters $\xi$ of our model are constant in each subdomain, in other words are considered under the form

$$
\xi(x):=\xi_{1} \chi_{\Omega_{1}}(x)+\xi_{2}\left(1-\chi_{\Omega_{1}}(x)\right),
$$

where $\chi_{\Omega_{i}}$ is the characteristic function of the sets $\Omega_{i}$ (equal to 1 if $x \in \Omega_{i}$ and to 0 otherwise) and $\xi_{1}, \xi_{2}$ are the mechanical parameters defined on $\Omega_{1}$ and $\Omega_{2}$.

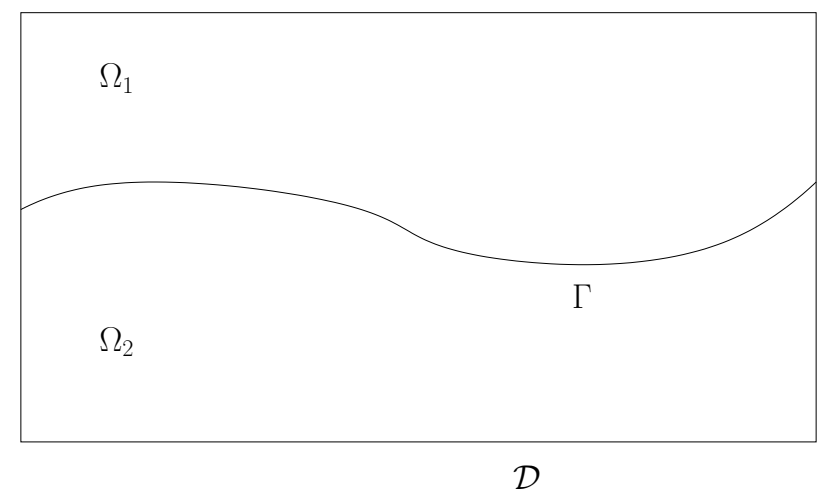

Figure 1: A domain $\mathcal{D}$ with two sub-domains separated by an interface $\Gamma$.

The mechanical unknowns of our model are the displacement field $u(t, x):\left[0, t_{f}\right] \times \mathcal{D} \rightarrow \mathbb{R}^{n}$, and the temperature field $T(t, x):\left[0, t_{f}\right] \times \mathcal{D} \rightarrow \mathbb{R}$. They follow a thermo-elastic time-evolution model, defined by the following equations.

- Heat equation:

$$
\begin{cases}\rho \frac{\partial T}{\partial t}-\operatorname{div}(\lambda \nabla T)=Q & \text { in }\left(0, t_{f}\right) \times \mathcal{D}, \\ (\lambda \nabla T) \cdot n=-\beta\left(T-T_{\text {init }}\right) & \text { on }\left(0, t_{f}\right) \times \partial \mathcal{D}_{F}, \\ T=T_{\text {init }} & \text { on }\left(0, t_{f}\right) \times \partial \mathcal{D}_{D}, \\ (\lambda \nabla T) \cdot n=g & \text { on }\left(0, t_{f}\right) \times \partial \mathcal{D}_{N}, \\ T(0)=T_{\text {init }} & \text { in } \mathcal{D},\end{cases}
$$

where $\lambda>0$ is the thermal conductivity coefficient, $\rho>0$ the product of the material density by the specific heat and $\beta>0$ the heat transfert coefficient. A Neumann boundary condition $g=g_{1} \chi_{\Omega_{1}}+g_{2}\left(1-\chi_{\Omega_{1}}\right)$ with $g_{1}, g_{2} \in L^{2}\left(\left(0, t_{f}\right), H^{1}(\mathcal{D})\right)$ is imposed on $\partial \mathcal{D}_{N}$ and a thermal body source $Q=Q_{1} \chi_{\Omega_{1}}+Q_{2}\left(1-\chi_{\Omega_{1}}\right)$ with $Q_{1}, Q_{2} \in L^{2}\left(\left(0, t_{f}\right), H^{1}(\mathcal{D})\right)$ is considered in $\mathcal{D}$. The initial and reference temperature is $T_{\text {init }} \in C\left(\left[0, t_{f}\right], L^{2}(\mathcal{D})\right) \cap$ $L^{2}\left(\left[0, t_{f}\right], H^{1}(\mathcal{D})\right)$. 
- Thermoelastic equilibrium system:

$$
\begin{cases}-\operatorname{div}(\sigma)=f & \text { in }\left(0, t_{f}\right) \times \mathcal{D} \\ \sigma=\sigma^{e l}+\sigma^{t h} & \text { in }\left(0, t_{f}\right) \times \mathcal{D} \\ \sigma^{e l}=A e(u) & \sigma^{t h}:=K\left(T-T_{\text {init }}\right) \mathbb{I}_{n} \\ \sigma \cdot n=0 & \text { on }\left(0, t_{f}\right) \times \partial \mathcal{D}_{N} \cup \partial \mathcal{D}_{F} \\ u=0 & \text { on }\left(0, t_{f}\right) \times \partial \mathcal{D}_{D}\end{cases}
$$

where $\sigma$ is the Cauchy stress tensor, decomposed as the sum of the elastic stress $\sigma^{e l}$ and the thermal stress $\sigma^{\text {th }}, A$ is the fourth-order elasticity tensor of an isotropic material with Young modulus $E>0$ and Poisson ratio $-1<\nu<1 / 2, \mathbb{I}_{n}$ the identity matrix, $\alpha$ the thermal expansion coefficient and

$$
K=-\frac{E \alpha}{1-2 \nu} .
$$

Recall that, under the usual assumptions of small displacements and small deformations, the strain tensor $e(u)$ is defined by $e(u)=\frac{1}{2}\left(\nabla u+(\nabla u)^{T}\right)$. The body forces (typically gravity) are $f=f_{1} \chi_{\Omega_{1}}+f_{2}\left(1-\chi_{\Omega_{1}}\right)$ with $f_{1}, f_{2} \in L^{2}\left(\left(0, t_{f}\right), H^{1}(\mathcal{D})^{n}\right)$. The associated variational formulations to the problems (2) and (3) are as follows. Find $T \in C\left(\left[0, t_{f}\right] ; L^{2}(\mathcal{D})\right) \cap L^{2}\left(\left(0, t_{f}\right) ; H^{1}(\mathcal{D})\right)$ such that $T(0)=T_{\text {init }}$ and for a.e. $0<t<t_{f}$, $T(t)=T_{\text {init }}$ on $\partial \mathcal{D}_{D}$ and for any $\varphi \in H_{D}^{1}(\mathcal{D})$

$\frac{d}{d t} \int_{\mathcal{D}} \rho T(t) \varphi d x+\int_{\mathcal{D}} \lambda \nabla T(t) \cdot \nabla \varphi d x+\int_{\partial \mathcal{D}_{F}} \beta\left(T(t)-T_{i n i t}\right) \varphi d s=\int_{\mathcal{D}} Q(t) \varphi d x+\int_{\partial \mathcal{D}_{N}} g(t) \varphi d s$.

Find $u \in L^{2}\left(\left(0, t_{f}\right) ; H_{D}^{1}(\mathcal{D})^{n}\right)$ such that for a.e. $t \in\left(0, t_{f}\right)$, for any $\varphi \in H_{D}^{1}(\mathcal{D})^{n}$

$$
\int_{\mathcal{D}}\left(A e(u(t))+K\left(T(t)-T_{i n i t}\right) \mathbb{I}_{n}\right): e(\varphi) d x=\int_{\mathcal{D}} f(t) \cdot \varphi d x
$$

where the symbol : denotes the full contraction (or scalar product) of square matrices.

Note that problem (2) can be solved independently of (3), and that problem (3) depends on the temperature field $T$, solution of (2). Under our assumptions, it is well known [29] that (2) admits a unique solution $T \in C\left(\left[0, t_{f}\right] ; L^{2}(\mathcal{D})\right) \cap L^{2}\left(\left(0, t_{f}\right) ; H^{1}(\mathcal{D})\right)$. This implies that $\operatorname{div} \sigma^{t h}$ is a forcing term that belong to the dual space of $L^{2}\left(\left(0, t_{f}\right) ; H_{D}^{1}(\mathcal{D})^{n}\right)$. Then, in turn $(3)$ admits a unique solution $u \in L^{2}\left(\left(0, t_{f}\right) ; H_{D}^{1}(\mathcal{D})^{n}\right)$.

Remark 2.1 It is only for the simplicity of exposition that Dirichlet boundary conditions are applied for both the temperature $T$ and the displacement $u$ on the same part $\partial \mathcal{D}_{D}$ of the boundary.

\subsection{The optimization problem}

In this section we optimize the position of the interface $\Gamma$ between two linear thermoelastic materials. Either $\Gamma$ is a smooth surface without boundary, included in the interior of $\mathcal{D}$, or $\Gamma$ is a smooth surface with a boundary which lies on the boundary of the domain, $\partial \Gamma \subset \partial \mathcal{D}$. The interface $\Gamma$ is the only optimisation variable ; the boundary $\partial \mathcal{D}$ and its subsets are not allowed to move.

Clearly, this is a simplification of the true optimization problem, described in the next section. We consider the following objective function

$$
J(\Gamma)=\int_{0}^{t_{f}} \int_{\mathcal{D}} j(u(\Gamma)) d x d t+\int_{0}^{t_{f}} \int_{\partial \mathcal{D}} l(u(\Gamma)) d s d t
$$


where $j$ and $l$ are two smooth functions with quadratic growth, and $u(\Gamma)$ is the elastic displacement solution of the model (2) and (3). Of course, (4) is just an example and many more choices are possible, for example, depending on the gradient $\nabla u$ or on the temperature $T$ too,

$$
J(\Gamma)=\int_{0}^{t_{f}} \int_{\mathcal{D}} j(u(\Gamma), \nabla u(\Gamma), T(\Gamma)) d x d t+\int_{0}^{t_{f}} \int_{\partial \mathcal{D}} l(u(\Gamma), T(\Gamma)) d s d t,
$$

or involving also an integral on the domain evaluated at the final time $t_{f}$. Our approach easily extends to such cases. Associated to the objective function (4) we introduce the following adjoint systems

$$
\begin{cases}-\operatorname{div}(A e(\eta))=-j^{\prime}(u) & \text { in }\left(0, t_{f}\right) \times \mathcal{D} \\ (A e(\eta)) \cdot n=-l^{\prime}(u) & \text { on }\left(0, t_{f}\right) \times \partial \mathcal{D}_{F} \cup \partial \mathcal{D}_{N}, \\ \eta=0 & \text { on }\left(0, t_{f}\right) \times \partial \mathcal{D}_{D},\end{cases}
$$

and

$$
\begin{cases}\rho \frac{\partial p}{\partial t}+\operatorname{div}(\lambda \nabla p)=K \operatorname{div}(\eta) & \text { in }\left(0, t_{f}\right) \times \mathcal{D}, \\ (\lambda \nabla p) \cdot n=-\beta p & \text { on }\left(0, t_{f}\right) \times \partial \mathcal{D}_{F}, \\ (\lambda \nabla p) \cdot n=0 & \text { on }\left(0, t_{f}\right) \times \partial \mathcal{D}_{N}, \\ p=0 & \text { on }\left(0, t_{f}\right) \times \partial \mathcal{D}_{D} \\ p\left(t_{f}\right)=0 & \text { in } \mathcal{D} .\end{cases}
$$

The variational formulations associated to the adjoint problems (6) and (7) are as follows. Find $p \in C\left(\left[0, t_{f}\right] ; L^{2}(\mathcal{D})\right) \cap L^{2}\left(\left(0, t_{f}\right) ; H_{D}^{1}(\mathcal{D})\right)$ such that $p\left(t_{f}\right)=0$ and for a.e. $0<t<t_{f}$, for any $\varphi \in H_{D}^{1}(\mathcal{D})$

$$
\frac{d}{d t} \int_{\mathcal{D}} \rho p(t) \varphi d x-\int_{\mathcal{D}} \lambda \nabla p(t) \cdot \nabla \varphi d x-\int_{\partial \mathcal{D}_{F}} \beta p(t) \varphi d s=\int_{\mathcal{D}} K \operatorname{div}(\eta(t)) \varphi d x .
$$

Find $\eta \in L^{2}\left(\left(0, t_{f}\right) ; H_{D}^{1}(\mathcal{D})^{n}\right)$ such that for a.e. $t \in\left(0, t_{f}\right)$, for any $\varphi \in H_{D}^{1}(\mathcal{D})^{n}$

$$
\int_{\mathcal{D}} A e(\eta(t)): e(\varphi) d x=-\int_{\mathcal{D}} j^{\prime}(u(t)) \cdot \varphi d x-\int_{\partial \mathcal{D}_{N} \cup \partial \mathcal{D}_{F}} l^{\prime}(u(t)) \cdot \varphi d s .
$$

Note that the coupling of (6) and (7) is the inverse of that of (2) and (3) in the sense that, here, one solves first the adjoint elasticity system (6) before solving in a second step the backward heat equation (7) with a final condition. It is well known that (6) admits a unique solution $\eta \in L^{2}\left(\left(0, t_{f}\right) ; H_{D}^{1}(\mathcal{D})^{n}\right)$ (time is just a parameter, playing no role), which implies that the source term $K \operatorname{div}(\eta)$ in $(7)$ belongs to the dual space of $L^{2}\left(\left(0, t_{f}\right) ; H_{D}^{1}(\mathcal{D})\right)$. Then, in turn $(7)$ admits a unique solution $T \in C\left(\left[0, t_{f}\right] ; L^{2}(\mathcal{D})\right) \cap L^{2}\left(\left(0, t_{f}\right) ; H^{1}(\mathcal{D})\right)$.

Although the notion of shape derivative is only introduced in the following section, let us say a few words about a classical difficulty in computing the shape derivative of the objective function (4) in the present setting. As explained in [1], [7], [10], [24], [33], the differentiation of the objective function $J(\Gamma)$ with respect to the position of the interface $\Gamma$ is a delicate topic for two reasons. First, the solution $(u, T)$ of the system (2) and (3) is not shape differentiable, in the usual sense, because the solution gradient has jumps through the interface. Second, even if this difficulty can be circumvented (by differentiating separately in the subdomains $\Omega_{1}$ and $\Omega_{2}$ ), the shape derivative features jumps of the solution gradient at the interface, which are always difficult to compute when using standard numerical methods on a fixed mesh where the interface is captured and not tracked. Therefore, it is much more convenient and numerically efficient to compute the shape derivative of a discrete version of this problem, as we now explain.

More precisely, in the rest of this section we replace the exact solution $(u, T, p, \eta)$ of systems (2), (3), (6) and (7) by its discret analogue $\left(u^{h}, T^{h}, p^{h}, \eta^{h}\right)$, solution of the following variational 
formulation (or rather semi-discrete because the time variable is not yet discretized). We introduce a finite-dimensional subspace $V_{h}$ of $H^{1}(\mathcal{D})$, which is independent of the interface $\Gamma$. For example, $V_{h}$ could be the finite-dimensional space of $\mathbb{P}^{1}$ Lagrange finite element associated to a given simplicial mesh $\mathcal{D}^{h}$ (with elements of maximal size $h$ ). We also defined its subset $V_{h 0}$ of all functions vanishing on the Dirichlet part of the boundary

$$
V_{h 0}=\left\{v \in V_{h} \text { such that } v=0 \text { on } \partial \mathcal{D}_{D}\right\} .
$$

The discrete variational formulations are: find $T^{h} \in C\left(\left[0, t_{f}\right] ; V_{h 0}\right)$ such that $T^{h}(0)=T_{\text {init }}$ and for $0<t<t_{f}$, for any $\varphi^{h} \in V_{h 0}$

$$
\begin{aligned}
\frac{d}{d t} \int_{\mathcal{D}} \rho T^{h}(t) \varphi^{h} d x+\int_{\mathcal{D}} \lambda \nabla T^{h}(t) \cdot \nabla \varphi^{h} d x & +\int_{\partial \mathcal{D}_{F}} \beta\left(T^{h}(t)-T_{\text {init }}\right) \varphi^{h} d s \\
& =\int_{\mathcal{D}} Q(t) \varphi^{h} d x+\int_{\partial \mathcal{D}_{N}} g(t) \varphi^{h} d s .
\end{aligned}
$$

Find $u^{h} \in L^{2}\left(\left(0, t_{f}\right) ; V_{h 0}^{n}\right)$ such that for a.e. $t \in\left(0, t_{f}\right)$, for any $\varphi^{h} \in V_{h 0}^{n}$

$$
\int_{\mathcal{D}}\left(A e\left(u^{h}(t)\right)+K\left(T^{h}(t)-T_{i n i t}\right) \mathbb{I}_{n}\right): e\left(\varphi^{h}\right) d x=\int_{\mathcal{D}} f(t) \cdot \varphi^{h} d x .
$$

Find $\eta^{h} \in L^{2}\left(\left(0, t_{f}\right) ; V_{h 0}^{n}\right)$ such that for $t \in\left(0, t_{f}\right)$, for any $\varphi^{h} \in V_{h 0}^{n}$

$$
\int_{\mathcal{D}} A e\left(\eta^{h}(t)\right): e\left(\varphi^{h}\right) d x=-\int_{\mathcal{D}} j^{\prime}\left(u^{h}(t)\right) \cdot \varphi^{h} d x-\int_{\partial \mathcal{D}_{N} \cup \partial \mathcal{D}_{F}} l^{\prime}\left(u^{h}(t)\right) \cdot \varphi^{h} d s,
$$

Find $p^{h} \in C\left(\left[0, t_{f}\right] ; V_{h 0}\right)$ such that $p^{h}\left(t_{f}\right)=0$ and for a.e. $0<t<t_{f}$, for any $\varphi^{h} \in V_{h 0}$

$$
\frac{d}{d t} \int_{\mathcal{D}} \rho p^{h}(t) \varphi^{h} d x-\int_{\mathcal{D}} \lambda \nabla p^{h}(t) \cdot \nabla \varphi^{h} d x=\int_{\mathcal{D}} K \operatorname{div}\left(\eta^{h}(t)\right) \varphi^{h} d x+\int_{\partial \mathcal{D}_{F}} \beta p^{h}(t) \varphi^{h} d s .
$$

The key point is that, under a mild assumption on the interface $\Gamma$, the discrete solution $\left(u^{h}, T^{h}\right)$ is now shape differentiable in the usual sense (see [1] for details).

Lemma 2.1 ([1]) Assume that the interface $\Gamma$ generically cuts the mesh $\mathcal{D}^{h}$, namely that it is never aligned with part of a face of any cell of the mesh. Let $\theta \in W^{1, \infty}(\mathcal{D}, \mathcal{D})$ be a displacement field for the interface $\Gamma$ such that $\theta \cdot n=0$ on $\partial \mathcal{D}$. Then, the discrete solution $\left(u^{h}, T^{h}\right)$ of (10) and (11) is differentiable with respect to the displacement $\theta$ of the interface $\Gamma$.

Because of the differentiability property of Lemma 2.1 and the deduced simplified formulas for shape derivatives, in the sequel, we shall always rely on the following assumption.

Assumption 2.2 In the present paper, we always replace the exact (continuous) solutions of the state and adjoint equations by their discrete approximations in finite dimensional subspaces. Furthermore, we assume that the interface $\Gamma$ generically cuts the mesh $\mathcal{D}^{h}$, namely that it is never aligned with part of a face of any cell of the mesh. Therefore, these discrete solutions are shape differentiable. Eventually, for simplicity in the presentation, we shall abuse notations and drop the superscript $h$ when considering these discrete solutions. 


\subsection{Shape derivative}

The setting of the previous section is slightly different from the classical framework of shape differentiation [12], [31], [34], [39], since it is the interface between two subdomains, rather than the exterior boundary of a single domain, which is the optimization variable. The main idea of the Hadamard method of shape differentiation is to parametrize the deformations of the interface $\Gamma$ of the subdomains $\Omega_{1}, \Omega_{2}$ by a vector field $\theta$ from $\mathcal{D}$ into $\mathcal{D}$, and then to differentiate with respect to $\theta$. In other words, for any $\theta \in W^{1, \infty}(\mathcal{D}, \mathcal{D})$ such that $\theta \cdot n=0$ on $\partial \mathcal{D}$ (the working domain $\mathcal{D}$ is fixed and not allowed to change with $\theta$ ), define

$$
\Gamma_{\theta}=(\operatorname{Id}+\theta) \Gamma \quad \text { and } \quad \Omega_{1, \theta}=(\operatorname{Id}+\theta) \Omega_{1},
$$

then shape derivatives are defined as derivatives with respect to $\theta$ at the origin. To establish the shape derivative for the objective function (4), the following standard lemma is needed.

Lemma 2.3 Let $\Omega_{1}$ be a smooth bounded open set included in $\mathcal{D}$ and $\theta \in W^{1, \infty}(\mathcal{D}, \mathcal{D})$ such that $\theta \cdot n=0$ on $\partial \mathcal{D}$. Let $f \in H^{1}(\mathcal{D})$ and $g \in H^{2}(\mathcal{D})$ be two given functions. Let $\Gamma$ be the interface between $\Omega_{1}$ and $\Omega_{2}=\mathcal{D} \backslash \Omega_{1}$ with a boundary $\partial \Gamma=\Gamma \cap \partial \mathcal{D}$. The shape derivatives of

$$
J_{1}(\Gamma)=\int_{\Omega_{1}} f d x \quad \text { and } \quad J_{2}(\Gamma)=\int_{\Gamma} g d s
$$

are

$$
J_{1}^{\prime}(\Gamma)(\theta)=\int_{\Gamma} f \theta \cdot n d s \quad \text { and } \quad J_{2}^{\prime}(\Gamma)(\theta)=\int_{\Gamma}\left(\frac{\partial g}{\partial n}+g H\right) \theta \cdot n d s+\int_{\partial \Gamma} g \theta \cdot \tau d L,
$$

respectively, where $n$ is the exterior unit vector normal to $\Omega_{1}, H$ is the mean curvature, $\tau$ is the unit vector tangent to $\Gamma$ and normal to $\partial \Gamma$, and $d L$ is the $(n-2)$-dimensional measure along $\partial \Gamma$.

Remark 2.2 Be careful that, by a slight abuse of notations, the same letter $n$ is used for the unit exterior normal to $\mathcal{D}$, when we assume that $\theta \cdot n=0$ on $\partial \mathcal{D}$, and for the unit exterior normal to $\Omega_{1}$ in the formula (14) for the shape derivatives. Note that, in (14), the term $\theta \cdot \tau$ on $\partial \Gamma=\Gamma \cap \partial \mathcal{D}$ is equal to $\theta_{t} \cdot \tau$ where $\theta_{t}=\theta-(\theta \cdot n) n$ is the tangential part of $\theta$ on $\partial \mathcal{D}$, since $\theta \cdot n=0$ on $\partial \mathcal{D}$.

Then, our main result is the computation of the shape derivative of the functional $J(\Gamma)$, defined by (4). Recall that, by Assumption 2.2, we always consider discrete solutions of the direct and adjoint problems, instead of the true continuous ones.

Theorem 2.4 Let $\theta \in W^{1, \infty}(\mathcal{D}, \mathcal{D})$ be a displacement field for the interface $\Gamma$ such that $\theta \cdot n=0$ on $\partial \mathcal{D}$. Under Assumption 2.2 the shape derivative of $J(\Gamma)$ is

$$
\begin{aligned}
J^{\prime}(\Gamma)(\theta)= & \int_{0}^{t_{f}} \int_{\Gamma}\left\{\left(\rho_{1}-\rho_{2}\right) \frac{\partial T}{\partial t} p+\left(\lambda_{1}-\lambda_{2}\right) \nabla T \cdot \nabla p-\left(Q_{1}-Q_{2}\right) p\right\} \theta \cdot n d s d t \\
& +\int_{0}^{t_{f}} \int_{\Gamma}\left\{\left(\left(A_{1}-A_{2}\right) e(u)+\left(K_{1}-K_{2}\right)\left(T-T_{i n i t}\right) \mathbb{I}_{n}\right): e(\eta)-\left(f_{1}-f_{2}\right) \cdot \eta\right\} \theta \cdot n d s d t \\
& +\int_{0}^{t_{f}} \int_{\partial \mathcal{D}_{F} \cap \partial \Gamma}\left(\beta_{1}-\beta_{2}\right)\left(T-T_{\text {init }}\right) p \theta \cdot \tau d L d t-\int_{0}^{t_{f}} \int_{\partial \mathcal{D}_{N} \cap \partial \Gamma}\left(g_{1}-g_{2}\right) p \theta \cdot \tau d L d t
\end{aligned}
$$

where $\eta$ and $p$ are the (discrete) solutions of the adjoint problems (12) and (13), $n$ is the normal unit vector to $\Gamma$ (oriented from $\Omega_{1}$ to $\Omega_{2}$ ), and $\tau$ is the unit vector tangent to $\Gamma$ and normal to $\partial \Gamma$, and $d L$ is the $(n-2)$-dimensional measure along $\partial \Gamma$. 
Proof Following the Lagrangian method of Céa [12], we introduce a Lagrangian depending on the interface $\Gamma$ and functions $\hat{T}, \hat{p} \in C\left(\left[0, t_{f}\right], V_{h}\right)$, and $\hat{u}, \hat{\eta} \in L^{2}\left(\left[0, t_{f}\right], V_{h 0}^{n}\right)$

$$
\begin{aligned}
\mathcal{L}(\Gamma, \hat{u}, \hat{\eta}, \hat{T}, \hat{p}) & =\int_{0}^{t_{f}} \int_{\mathcal{D}} j(\hat{u}) d x d t+\int_{0}^{t_{f}} \int_{\partial \mathcal{D}} l(\hat{u}) d s d t+\int_{0}^{t_{f}} \int_{\mathcal{D}}\left\{\rho \frac{\partial \hat{T}}{\partial t} \hat{p}+\lambda \nabla \hat{T} \cdot \nabla \hat{p}-Q \hat{p}\right\} d x d t \\
& +\int_{0}^{t_{f}} \int_{\partial \mathcal{D}_{F}} \beta\left(\hat{T}-T_{\text {init }}\right) \hat{p} d s d t-\int_{0}^{t_{f}} \int_{\partial \mathcal{D}_{D}}\left\{(\lambda \nabla \hat{T}) \cdot n \hat{p}+(\lambda \nabla \hat{p}) \cdot n\left(\hat{T}-T_{\text {init }}\right)\right\} d s d t \\
& -\int_{0}^{t_{f}} \int_{\partial \mathcal{D}_{N}} g \hat{p} d s d t+\int_{\mathcal{D}} \rho\left(\hat{T}(0)-T_{\text {init }}\right) \hat{p}(0) d x \\
& +\int_{0}^{t_{f}} \int_{\mathcal{D}}\left\{\left(A e(\hat{u})+K\left(\hat{T}-T_{\text {init }}\right) \mathbb{I}_{n}\right): e(\hat{\eta})-f \cdot \hat{\eta}\right\} d x d t .
\end{aligned}
$$

Differentiating $\mathcal{L}$ with respect to $\hat{\eta}$ in the direction of a test function $\varphi \in L^{2}\left(\left[0, t_{f}\right], V_{h 0}^{n}\right)$, and making this derivative equal to zero at the optimal point $(T, u, \eta, p)$ we obtain

$$
\int_{0}^{t_{f}} \int_{\mathcal{D}}\left\{\left(A e(u)+K\left(T-T_{\text {init }}\right) \mathbb{I}_{n}\right): e(\varphi)-f \cdot \varphi\right\} d x d t=0
$$

which is nothing else than the (discrete) variational formulation of the thermo-elastic system (3). Similarly, the partial derivative of $\mathcal{L}$ with respect to $\hat{p}$ in the direction of a test function $\varphi \in C\left(\left[0, t_{f}\right], V_{h}\right)$, equal to zero at the optimal point $(T, u, \eta, p)$, gives

$$
\begin{aligned}
& \int_{0}^{t_{f}} \int_{\mathcal{D}}\left\{\rho \frac{\partial T}{\partial t} \varphi+\lambda \nabla T \cdot \nabla \varphi-Q \varphi\right\} d x d t+\int_{0}^{t_{f}} \int_{\partial \mathcal{D}_{F}} \beta\left(T-T_{\text {init }}\right) \varphi d s d t \\
& -\int_{0}^{t_{f}} \int_{\partial \mathcal{D}_{D}}\left\{(\lambda \nabla T) \cdot n \varphi+(\lambda \nabla \varphi) \cdot n\left(T-T_{\text {init }}\right)\right\} d s d t \\
& -\int_{0}^{t_{f}} \int_{\partial \mathcal{D}_{N}} g \varphi d s d t+\int_{\mathcal{D}} \rho\left(T(0)-T_{\text {init }}\right) \varphi(0) d x=0 .
\end{aligned}
$$

First, restricting ourselves to compactly supported test functions $\phi \in C_{c}^{\infty}\left(\mathcal{D} \times\left(0, t_{f}\right)\right)$, (15) yields the heat equation in (2). Second, taking into account this heat equation, after integration by parts for a general test function, (15) reduces to

$$
\begin{aligned}
& \int_{0}^{t_{f}} \int_{\partial \mathcal{D}_{F}}(\lambda \nabla T) \cdot n \varphi+\beta\left(T-T_{\text {init }}\right) \varphi d s d t-\int_{0}^{t_{f}} \int_{\partial \mathcal{D}_{D}}(\lambda \nabla \varphi) \cdot n\left(T-T_{\text {init }}\right) d s d t \\
+ & \int_{0}^{t_{f}} \int_{\partial \mathcal{D}_{N}}((\lambda \nabla T) \cdot n-g) \varphi d s d t+\int_{\mathcal{D}} \rho\left(T(0)-T_{\text {init }}\right) \varphi(0) d x=0 .
\end{aligned}
$$

Varying $\varphi$ leads to the initial condition $T(0)=T_{\text {init }}$ in $\mathcal{D}$, as well as the various boundary conditions.

To find the adjoint systems, we now differentiate $\mathcal{L}$ with respect to the primal displacement variable $\hat{u}$ in the direction of a test function $\varphi \in L^{2}\left(\left[0, t_{f}\right], V_{h 0}^{n}\right)$ to obtain at optimality

$$
\int_{0}^{t_{f}} \int_{\mathcal{D}} j^{\prime}(u) \cdot \varphi d x d t+\int_{0}^{t_{f}} \int_{\partial \mathcal{D}} l^{\prime}(u) \cdot \varphi d s d t+\int_{0}^{t_{f}} \int_{\mathcal{D}} A e(\varphi): e(\eta) d x d t=0
$$

which is nothing else than the (discrete) variational formulation (12) of the thermo-elastic adjoint system. Then, we differentiate $\mathcal{L}$ with respect to the primal temperature variable $\hat{T}$ in the 
direction of a test function $\varphi \in C\left(\left[0, t_{f}\right], V_{h}\right)$ which, at optimality, leads to

$$
\begin{gathered}
\int_{0}^{t_{f}} \int_{\mathcal{D}}\left\{\rho \frac{\partial \varphi}{\partial t} p+\lambda \nabla \varphi \cdot \nabla p+K \operatorname{div}(\eta) \varphi\right\} d x d t+\int_{0}^{t_{f}} \int_{\partial \mathcal{D}_{F}} \beta \varphi p d s d t \\
-\int_{0}^{t_{f}} \int_{\partial \mathcal{D}_{D}}\{(\lambda \nabla \varphi) \cdot n p+(\lambda \nabla p) \cdot n \varphi\} d s d t+\int_{\mathcal{D}} \rho \varphi(0) p(0) d x=0 .
\end{gathered}
$$

An integration by parts, both in time and space variables, leads to

$$
\begin{aligned}
& \int_{\mathcal{D}} \rho \varphi\left(t_{f}\right) p\left(t_{f}\right) d x-\int_{0}^{t_{f}} \int_{\mathcal{D}}\left\{\rho \frac{\partial p}{\partial t}+\operatorname{div}(\lambda \nabla p)-K \operatorname{div}(\eta)\right\} \varphi d x d t \\
& \quad-\int_{0}^{t_{f}} \int_{\partial \mathcal{D}_{D}}(\lambda \nabla \varphi) \cdot n p d s d t+\int_{0}^{t_{f}} \int_{\partial \mathcal{D}_{F}}\{(\lambda \nabla p) \cdot n+\beta p\} \varphi d s d t \\
& \quad+\int_{0}^{t_{f}} \int_{\partial \mathcal{D}_{N}}(\lambda \nabla p) \cdot n \varphi d s d t=0 .
\end{aligned}
$$

Varying the test function $\varphi$ in (16) leads to the thermal adjoint equation (7), its final condition at $t_{f}$ and boundary conditions.

It remains to compute the shape derivative. By Lemma 2.1, under Assumption 2.2 that the interface $\Gamma$ is not aligned with part of a face of any cell of the mesh, the discrete solution $(u, T)$ (omitting $h$ ) is shape differentiable in the usual sense. Then, it is a classical result [6], [12] that the shape derivative of the objective function $J$ is just the partial derivative of the Lagrangian $\mathcal{L}$

$$
J^{\prime}(\Gamma)(\theta)=\frac{\partial \mathcal{L}}{\partial \Gamma}(\Gamma, u, T, \eta, p)(\theta) .
$$

Thus, we apply Lemma 2.3 to the Lagrangian. The normal vector to $\Omega_{1}$ is $n_{1}$, the normal vector to $\Omega_{2}$ is $n_{2}$ and $n_{1}=-n_{2}$. Recall that only $\Gamma$ is free to move. We start by differentiating volume integrals. Since $u$ is continuous through the interface $\Gamma$, the following derivative cancels

$$
\begin{aligned}
\frac{\partial}{\partial \Gamma}\left(\int_{0}^{t_{f}} \int_{\mathcal{D}} j(u) d x d t\right)(\theta) & =\frac{\partial}{\partial \Gamma}\left(\int_{0}^{t_{f}} \int_{\Omega_{1}} j(u) d x d t\right)(\theta)+\frac{\partial}{\partial \Gamma}\left(\int_{0}^{t_{f}} \int_{\Omega_{2}} j(u) d x d t\right)(\theta) \\
& =\int_{0}^{t_{f}} \int_{\Gamma} j(u) \theta \cdot n_{1} d s d t+\int_{0}^{t_{f}} \int_{\Gamma} j(u) \theta \cdot n_{2} d s d t=0 .
\end{aligned}
$$

Other volumic terms in the Lagrangian have non-zero derivatives since the material coefficients are discontinuous at the interface. For example,

$$
\begin{aligned}
\frac{\partial}{\partial \Gamma}\left(\int_{0}^{t_{f}} \int_{\mathcal{D}} A e(u): e(\eta) d x d t\right)(\theta)= & \frac{\partial}{\partial \Gamma}\left(\int_{0}^{t_{f}} \int_{\Omega_{1}} A_{1} e(u): e(\eta) d x d t\right)(\theta) \\
& +\frac{\partial}{\partial \Gamma}\left(\int_{0}^{t_{f}} \int_{\Omega_{2}} A_{2} e(u): e(\eta) d x d t\right)(\theta) \\
= & \int_{0}^{t_{f}} \int_{\Gamma} \theta \cdot n_{1}\left(A_{1}-A_{2}\right) e(u): e(\eta) d s d t .
\end{aligned}
$$

In the above result we strongly rely on Assumption 2.2 that $(u, T, \eta, p)$ are the discrete solutions which thus have no discontinuity, as well as their derivatives, through the interface $\Gamma$.

We now turn to surface integrals. According to Lemma 2.3, $\tau_{1}=-\tau_{2}$, where $\tau_{1}$ is the normal vector to $\partial \Gamma$ and $n_{1}$. Since $u$ and its derivative are continuous through the interface $\Gamma$, the 
following derivative cancels

$$
\begin{aligned}
\frac{\partial}{\partial \Gamma}\left(\int_{0}^{t_{f}} \int_{\partial \mathcal{D}} l(u) d s d t\right)(\theta) & =\frac{\partial}{\partial \Gamma}\left(\int_{0}^{t_{f}} \int_{\partial \Omega_{1} \cap \partial \mathcal{D}} l(u) d s d t\right)(\theta)+\frac{\partial}{\partial \Gamma}\left(\int_{0}^{t_{f}} \int_{\partial \Omega_{2} \cap \partial \mathcal{D}} l(u) d s d t\right)(\theta) \\
& =\int_{0}^{t_{f}} \int_{\partial \Gamma}\left(\frac{\partial l(u)}{\partial n}+l(u) H\right) \theta \cdot n_{1} d s+\int_{0}^{t_{f}} \int_{\partial \Gamma} l(u) \theta \cdot \tau_{1} d L \\
& +\int_{0}^{t_{f}} \int_{\partial \Gamma}\left(\frac{\partial l(u)}{\partial n}+l(u) H\right) \theta \cdot n_{2} d s+\int_{0}^{t_{f}} \int_{\partial \Gamma} l(u) \theta \cdot \tau_{2} d L=0 .
\end{aligned}
$$

In the same way, we treat all surface integrals and the only non-zero terms come from the presence of discontinuous coefficients. For example,

$$
\begin{aligned}
\frac{\partial}{\partial \Gamma}\left(\int_{0}^{t_{f}} \int_{\partial \mathcal{D}_{F}} \beta\left(T-T_{\text {init }}\right) p d s d t\right)(\theta) & =\frac{\partial}{\partial \Gamma}\left(\int_{0}^{t_{f}} \int_{\partial \Omega_{1} \cap \partial \mathcal{D}_{F}} \beta_{1}\left(T-T_{\text {init }}\right) p d s d t\right)(\theta) \\
& +\frac{\partial}{\partial \Gamma}\left(\int_{0}^{t_{f}} \int_{\partial \Omega_{2} \cap \partial \mathcal{D}_{F}} \beta_{2}\left(T-T_{\text {init }}\right) p d s d t\right)(\theta) \\
& =\int_{0}^{t_{f}} \int_{\partial \Gamma \cap \partial \mathcal{D}_{F}}\left(\beta_{1}-\beta_{2}\right)\left(T-T_{\text {init }}\right) p \theta \cdot \tau_{1} d L
\end{aligned}
$$

Note that the term in the Lagrangian, corresponding to the initial condition, gives no contribution to the shape derivative because, indeed, the temperature $T$ satisfies this initial condition. This yields the desired result.

\subsection{A time discretization procedure}

In the previous section, in order to simplify the calculus of the shape derivative, discrete solutions (in space) were considered. Now, for computational reasons, the solutions should also be discretized in time. The goal of the present section is thus to give a consistent time discretization of the direct and adjoint solutions, of the objective function and its shape derivative. The time interval $\left(0, t_{f}\right)$ is discretized in $N$ subintervals $\left(t_{j-1}, t_{j}\right)$ of equal length $\Delta t=t_{j}-t_{j-1}=t_{f} / N$ such that

$$
0=t_{0}<t_{1}<\ldots<t_{j}<\ldots<t_{N}=t_{f} .
$$

The direct problem (2) and (3) is discretized by a backward (implicit) Euler scheme with respect to the time variable. For $1 \leq j \leq N$, on each subinterval $\left(t_{j-1}, t_{j}\right)$ the discrete time evolution $\left(u_{j}, T_{j}\right)$ is defined as the solution of the following system of equations. The initial temperature is $T_{j=0}=T_{0}=T_{\text {init }}$ in $\mathcal{D}$.

- Heat equation:

$$
\begin{cases}\rho \frac{T_{j}-T_{j-1}}{\Delta t}-\operatorname{div}\left(\lambda \nabla T_{j}\right)=Q_{j} & \text { in } \mathcal{D} \\ \left(\lambda \nabla T_{j}\right) \cdot n=-\beta\left(T_{j}-T_{\text {init }}\right) & \text { on } \partial \mathcal{D}_{F} \\ T_{j}=T_{\text {init }} & \text { on } \partial \mathcal{D}_{D} \\ \left(\lambda \nabla T_{j}\right) \cdot n=g_{j} & \text { on } \partial \mathcal{D}_{N} .\end{cases}
$$

- Thermoelastic equilibrium system:

$$
\begin{cases}-\operatorname{div}\left(\sigma_{j}\right)=f_{j} & \text { in } \mathcal{D} \\ \sigma_{j}=\sigma_{j}^{e l}+\sigma_{j}^{t h} & \text { in } \mathcal{D} \\ \sigma_{j}^{e l}=A e\left(u_{j}\right) & \sigma_{j}^{t h}:=K\left(T_{j}-T_{\text {init }}\right) \mathbb{I}_{n} \\ \sigma_{j} \cdot n=0 & \text { on } \partial \mathcal{D}_{N} \cup \partial \mathcal{D}_{F} \\ u_{j}=0 & \text { on } \partial \mathcal{D}_{D}\end{cases}
$$


The discrete time objective function is given by a simple piecewise constant interpolation

$$
J_{\Delta t}(\Gamma)=\sum_{j=1}^{N} \Delta t \int_{\mathcal{D}} j\left(u_{j}(\Gamma)\right) d x+\sum_{j=1}^{N} \Delta t \int_{\partial \mathcal{D}} l\left(u_{j}(\Gamma)\right) d s .
$$

To the discrete time objective function (19) are associated the following adjoint systems. The thermo-elastic adjoint problem is, for any $1 \leq j \leq N$,

$$
\begin{cases}-\operatorname{div}\left(A e\left(\eta_{j}\right)\right)=-j^{\prime}\left(u_{j}\right) & \text { in } \mathcal{D} \\ \left(A e\left(\eta_{j}\right)\right) \cdot n=-l^{\prime}\left(u_{j}\right) & \text { on } \partial \mathcal{D}_{F} \cup \partial \mathcal{D}_{N} \\ \eta_{j}=0 & \text { on } \partial \mathcal{D}_{D}\end{cases}
$$

The heat adjoint equation is, for $1 \leq j \leq N-1$,

$$
\begin{cases}\rho \frac{p_{j+1}-p_{j}}{\Delta t}+\operatorname{div}\left(\lambda \nabla p_{j}\right)=K \operatorname{div}\left(\eta_{j}\right) & \text { in } \mathcal{D} \\ \left(\lambda \nabla p_{j}\right) \cdot n=-\beta p_{j} & \text { on } \partial \mathcal{D}_{F}, \\ \left(\lambda \nabla p_{j}\right) \cdot n=0 & \text { on } \partial \mathcal{D}_{N} \\ p_{j}=0 & \text { on } \partial \mathcal{D}_{D}\end{cases}
$$

and the "final condition" for $j=N$

$$
\begin{cases}\rho p_{N}-\Delta t \operatorname{div}\left(\lambda \nabla p_{N}\right)=-\Delta t K \operatorname{div}\left(\eta_{N}\right) & \text { in } \mathcal{D} \\ \left(\lambda \nabla p_{N}\right) \cdot n=-\beta p_{N} & \text { on } \partial \mathcal{D}_{F}, \\ \left(\lambda \nabla p_{N}\right) \cdot n=0 & \text { on } \partial \mathcal{D}_{N}, \\ p_{N}=0 & \text { on } \partial \mathcal{D}_{D} .\end{cases}
$$

Remark 2.3 Note that one can easily show that $\left\|p_{N}\right\|_{L^{2}(\mathcal{D})} \leq C \Delta t$ which is consistant with the fact that the continuous adjoint $p(t)$ vanishes at the final time $t_{f}$.

The discrete time version of the shape derivative of Theorem 2.4 is the following result.

Theorem 2.5 Let $\theta \in W^{1, \infty}(\mathcal{D}, \mathcal{D})$ be such that $\theta \cdot n=0$ on $\partial \mathcal{D}$. Under Assumption 2.2 the shape derivative of (19) is

$$
\begin{aligned}
J_{\Delta t}^{\prime}(\Gamma)(\theta) & =\Delta t \sum_{j=1}^{N} \int_{\Gamma} \theta \cdot n\left\{\left(\rho_{1}-\rho_{2}\right) \frac{T_{j}-T_{j-1}}{\Delta t} p_{j}+\left(\lambda_{1}-\lambda_{2}\right) \nabla T_{j} \cdot \nabla p_{j}-\left(Q_{j}^{1}-Q_{j}^{2}\right) p_{j}\right\} d s \\
& +\Delta t \sum_{j=1}^{N} \int_{\Gamma} \theta \cdot n\left\{\left(\left(A_{1}-A_{2}\right) e\left(u_{j}\right)+\left(K_{1}-K_{2}\right)\left(T_{j}-T_{i n i t}\right) \mathbb{I}_{n}\right): e\left(\eta_{j}\right)-\left(f_{j}^{1}-f_{j}^{2}\right) \cdot \eta_{j}\right\} d s \\
& +\Delta t \sum_{j=1}^{N} \int_{\partial \mathcal{D}_{F} \cap \partial \Gamma}\left(\beta_{1}-\beta_{2}\right)\left(T_{j}-T_{i n i t}\right) p_{j} \theta \cdot \tau d L-\Delta t \sum_{j=1}^{N} \int_{\partial \mathcal{D}_{N} \cap \partial \Gamma}\left(g_{j}^{1}-g_{j}^{2}\right) p_{j} \theta \cdot \tau d L
\end{aligned}
$$

where $\eta_{j}$ and $p_{j}$ are the (discrete) solutions of the adjoint problems (20),(22) and (21), $n$ is the normal unit vector to $\Gamma$ (oriented from $\Omega_{1}$ to $\Omega_{2}$ ), and $\tau$ is the unit vector tangent to $\Gamma$ and normal to $\partial \Gamma$, and $d L$ is the $(n-2)$-dimensional measure along $\partial \Gamma$.

Proof For $1 \leq j \leq N$, let $\hat{T}_{j}, \hat{p}_{j} \in V_{h} \times V_{h 0}, \hat{u}_{j}, \hat{\eta}_{j} \in V_{h 0}^{n}$. By convention $\hat{T}_{0}=T_{\text {init }}$. We consider 
the Lagrangian

$$
\begin{aligned}
\mathcal{L}\left(\Gamma, \hat{u}_{j}, \hat{\eta}_{j}, \hat{T}_{j}, \hat{p}_{j}\right) & =\Delta t \sum_{j=1}^{N} \int_{\mathcal{D}} j\left(\hat{u}_{j}\right) d x+\Delta t \sum_{j=1}^{N} \int_{\partial \mathcal{D}} l\left(\hat{u}_{j}\right) d s \\
& +\Delta t \sum_{j=1}^{N} \int_{\mathcal{D}}\left\{\rho \frac{\hat{T}_{j}-\hat{T}_{j-1}}{\Delta t} \hat{p}_{j}+\lambda \nabla \hat{T}_{j} \cdot \nabla \hat{p}_{j}-Q_{j} \hat{p}_{j}\right\} d x \\
& +\Delta t \sum_{j=1}^{N} \int_{\partial \mathcal{D}_{F}} \beta\left(\hat{T}_{j}-T_{i n i t}\right) \hat{p}_{j} d s-\Delta t \sum_{j=1}^{N} \int_{\partial \mathcal{D}_{N}} g \hat{p}_{j} d s \\
& -\Delta t \sum_{j=1}^{N} \int_{\partial \mathcal{D}_{D}}\left\{\left(\lambda \nabla \hat{T}_{j}\right) \cdot n \hat{p}_{j}+\left(\lambda \nabla \hat{p}_{j}\right) \cdot n\left(\hat{T}_{j}-T_{\text {init }}\right)\right\} d s \\
& +\Delta t \sum_{j=1}^{N} \int_{\mathcal{D}}\left\{\left(\operatorname{Ae}\left(\hat{u}_{j}\right)+K\left(\hat{T}_{j}-T_{\text {init }}\right) \mathbb{I}_{n}\right): e\left(\hat{\eta}_{j}\right)-f \cdot \hat{\eta}_{j}\right\} d x .
\end{aligned}
$$

The proof follows the ideas of Theorem 2.4. For the sake of brevity, we treat only the differentiation with respect to the primal temperature variable $\hat{T}_{j}$ which yields a retrograde scheme in time for the associated dual variables $\hat{p}_{j}$.

First differentiate $\mathcal{L}$ with respect to $\hat{T}_{N}$ in the direction of a test function $\varphi \in V_{h}$ to get at optimality

$$
\begin{aligned}
& \int_{\mathcal{D}}\left\{\rho \frac{\varphi p_{N}}{\Delta t}+\lambda \nabla \varphi \cdot \nabla p_{N}+K \operatorname{div}\left(\eta_{N}\right) \varphi\right\} d x+\int_{\partial \mathcal{D}_{F}} \beta \varphi p_{N} d s \\
- & \int_{\partial \mathcal{D}_{D}}\left\{(\lambda \nabla \varphi) \cdot n p_{N}+\left(\lambda \nabla p_{N}\right) \cdot n \varphi\right\} d s=0 .
\end{aligned}
$$

from which we deduce system $(22)$ for $p_{N}$. Second, differentiate $\mathcal{L}$ with respect to $\hat{T}_{j}$, for $1 \leq j \leq N-1$, in the direction of a test function $\varphi \in V_{h}$ to obtain

$$
\begin{aligned}
& \int_{\mathcal{D}}\left\{\rho \frac{p_{j+1}-p_{j}}{\Delta t}+\lambda \nabla \varphi \cdot \nabla p_{j}+K \operatorname{div}\left(\eta_{j}\right) \varphi\right\} d x+\int_{\partial \mathcal{D}_{F}} \beta \varphi p_{j} d s \\
- & \int_{\partial \mathcal{D}_{D}}\left\{(\lambda \nabla \varphi) \cdot n p_{j}+\left(\lambda \nabla p_{j}\right) \cdot n \varphi\right\} d s=0 .
\end{aligned}
$$

from which we deduce system (21) for $p_{j}$. The other adjoint equations and the shape derivative are obtained by similar arguments.

\section{A shape optimization problem in additive manufacturing}

We now generalize the model of Section 2 to take into account the layer by layer construction process which is at stake in additive manufacturing. More precisely, we follow an approach initiated in [2], [3] for structural optimization. The equations are still the same as in Section 2 but we add the fact that the solid part is evolving in time during the building process.

\subsection{A layer by layer model for additive manufacturing}

We specify our notations to the case of additive manufacturing. The build chamber $\mathcal{D}$ is now a cylindrical domain $\mathcal{D}=S \times(0, H)$ where $H>0$ is its height and its cross-section $S \subset \mathbb{R}^{d-1}$ is a 


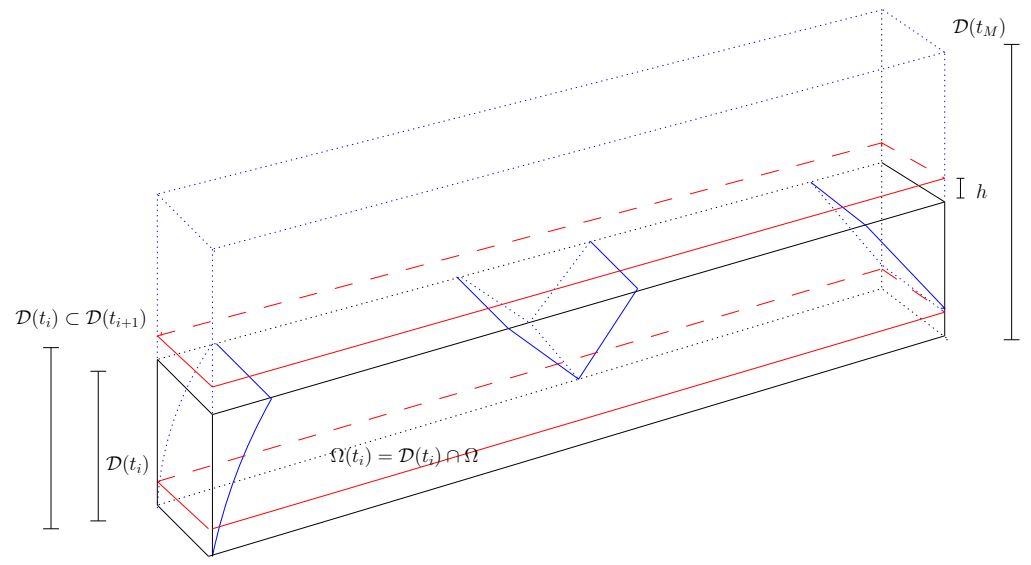

Figure 2: Construction layer by layer. Intermediate domain $\mathcal{D}\left(t_{i}\right)$ which is a mixture of powder and solid.

smooth simply connected open set: typically, a rectangle $S=\prod_{k=1}^{d-1}\left(0, L_{k}\right)$. By convention, the vertical direction is the building direction. The build chamber $\mathcal{D}$ is divided into $M$ horizontal layers of equal thickness $h=H / M$. Let $\left(t_{i}\right)_{0 \leq i \leq M}$ be a sequence of increasing time with $t_{0}=0$ and $t_{M}=t_{f}$ such that at each time $t_{i-1}(i \geq 1)$ a new layer is added (in a negligible amount of time). Note that the index $M$ has nothing to do with the index $N$ in the previous Section 2 . We define the intermediate domain $\mathcal{D}\left(t_{i}\right)=S \times(0, i h)$ (see Figure 2), satisfying

$$
\mathcal{D}\left(t_{1}\right) \subset \ldots \subset \mathcal{D}\left(t_{i}\right) \subset \ldots \subset \mathcal{D}\left(t_{f}\right) \equiv \mathcal{D} .
$$

Each $\mathcal{D}\left(t_{i+1}\right)$ is obtained from the previous $\mathcal{D}\left(t_{i}\right)$ by adding a layer $\mathcal{L}_{i+1}=S \times(i h,(i+1) h)$ (see Figure 4$)$. At time $t_{i}$ the composition of the previous subdomain $\mathcal{D}\left(t_{i}\right)$ is known and fixed: it is a mixture of metallic powder and solid. If $\Omega$ is the final shape after construction in $\mathcal{D}$ (see Figure 3), then the subdomain $\mathcal{P}:=\mathcal{D} \backslash \bar{\Omega}$ is occupied by powder, $\Omega\left(t_{i}\right):=\Omega \cap \mathcal{D}\left(t_{i}\right)$ is the intermediate solid shape and $\mathcal{P}\left(t_{i}\right):=\mathcal{P} \cap \mathcal{D}\left(t_{i}\right)$ is the intermediate powder subdomain in $\mathcal{D}\left(t_{i}\right)$. On the contrary, the composition of the layer $\mathcal{L}_{i+1}$ is going to change between times $t_{i}$ and $t_{i+1}$. Initially, $\mathcal{L}_{i+1}$ is made only of powder but, after the heat source term (a laser or electron beam) has passed, it is made partly of powder and partly of solid. The melting and solidification process is completely neglected: powder is immediately changed into solid upon heating by the source term.

Remark 3.1 By comparison with the previous section where the domain $\mathcal{D}$ was decomposed into two sub-domains $\Omega_{1}$ and $\Omega_{2}$, now each intermediate domain $\mathcal{D}\left(t_{i}\right)$, after completion at time $t_{i}$, is decomposed into two sub-domains $\Omega\left(t_{i}\right)$ and $\mathcal{P}\left(t_{i}\right)$, occupied by two different materials, solid and powder, respectively. In other words, $\Omega\left(t_{i}\right)$ plays the role of $\Omega_{1}$ and $\mathcal{P}\left(t_{i}\right)$ that of $\Omega_{2}$.

To define the material properties in the layer $\mathcal{L}_{i+1}$, we first need to define the heat source term, which is

$$
Q(x, t):=q \chi_{\text {beam }}(t, x) \chi_{\Omega}(x)
$$

where $q \in \mathbb{R}$ is a fixed value, $\chi_{\Omega}(x)$ is the characteristic function of the shape $\Omega$ and $\chi_{\text {beam }}(t, x)$ is the characteristic function of the beam spot, travelling with time along the beam path (see Figure 3). More precisely, for $t_{i}<t<t_{i+1}, \chi_{\text {beam }}$ is defined by

$$
\chi_{\text {beam }}(t, x)=\chi_{\text {spot }}\left(x^{\prime}-x_{i+1}^{\prime}(t), x_{d}-i h\right)
$$




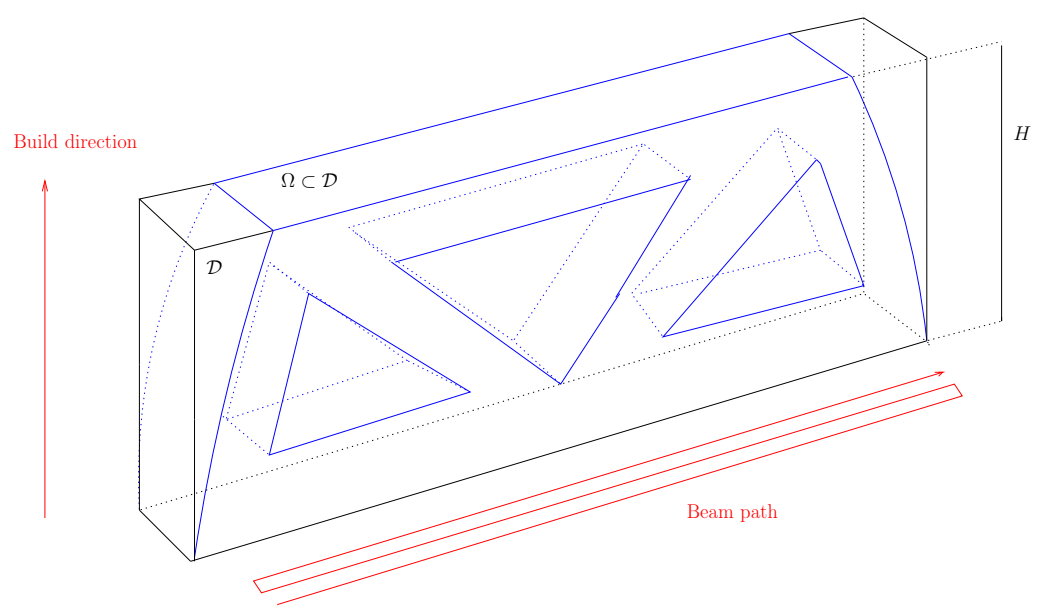

Figure 3: A final shape $\Omega$ in the build chamber $\mathcal{D}$.

where $x=\left(x^{\prime}, x_{d}\right) \in S \times(0, H), x_{i+1}^{\prime}(t)$ is the trajectory of the beam spot in the cross-section $S$, for $t_{i}<t<t_{i+1}$, and $\chi_{\text {spot }}(x)$ is the characteristic function of the beam spot, assumed to have compact support in one layer $S \times(0, h)$. We now define the characteristic function of that part of the layer $\mathcal{L}_{i+1}$ already built at time $t$, for $t_{i}<t<t_{i+1}$,

$$
\chi_{\mathcal{L}}(t, x)= \begin{cases}\chi_{\Omega}(x) & \text { if } x^{\prime} \text { has already been reached by the trajectory } x_{i+1}^{\prime}(t) \text { at time } t \\ 0 & \text { otherwise. }\end{cases}
$$

In other words, the characteristic function $\chi_{\mathcal{L}}(t, x)$ is the cumulated characteristic function of the heat source term.

Remark 3.2 The trajectory $x_{i}^{\prime}(t)$ of the beam spot in the top layer $\mathcal{L}_{i}$ is an important data of the building process and it can follow a quite complicated path [8], [16]. In particular, the speed (i.e. its time derivative) is a crucial parameter of additive manufacturing. However, in numerical practice, we content ourselves with simple trajectories, which are straight lines, and with a speed which corresponds to moving the source term from one macro-cell to the next one at each time step (see Figure 4 and Section 5.3 for details).

It is now possible to define the time dependent material property $\xi(t, x)$, which is a piecewise constant function. Recall that, between times $t_{i}$ and $t_{i+1}$, only the last layer $\mathcal{L}_{i+1}$ in domain $\mathcal{D}\left(t_{i+1}\right)$ has changing material properties. Thus, for $t_{i}<t<t_{i+1}$,

$$
\xi(t, x)= \begin{cases}\xi_{\Omega} \chi_{\Omega}(x)+\xi_{\mathcal{P}} \chi_{\mathcal{P}}(x) & \text { if } x \in \mathcal{D}\left(t_{i}\right), \\ \xi_{\Omega} \chi_{\mathcal{L}}(t, x)+\xi_{\mathcal{P}}\left(1-\chi_{\mathcal{L}}(t, x)\right) & \text { if } x \in \mathcal{L}_{i+1}\end{cases}
$$

All material properties being defined by (23), the layer by layer model for additive manufacturing is defined as an ordered sequence of problems posed in the intermediate domains $\mathcal{D}\left(t_{i}\right)$ for the time interval $\left(t_{i-1}, t_{i}\right)$. For $1 \leq i \leq M,\left(u_{i}, T_{i}\right)$ are the solutions of the heat equation

$$
\begin{cases}\rho \frac{\partial T_{i}}{\partial t}-\operatorname{div}\left(\lambda \nabla T_{i}\right)=Q & \text { in }\left(t_{i-1}, t_{i}\right) \times \mathcal{D}\left(t_{i}\right), \\ \left(\lambda \nabla T_{i}\right) \cdot n=-\beta\left(T_{i}-T_{\text {init }}\right) & \text { on }\left(t_{i-1}, t_{i}\right) \times \partial \mathcal{D}\left(t_{i}\right)_{N}, \\ T_{i}=T_{i n i t} & \text { on }\left(t_{i-1}, t_{i}\right) \times \partial \mathcal{D}\left(t_{i}\right)_{D} \\ T_{i}\left(t_{i-1}\right)=\tilde{T}_{i-1} & \text { in } \mathcal{D}\left(t_{i}\right),\end{cases}
$$




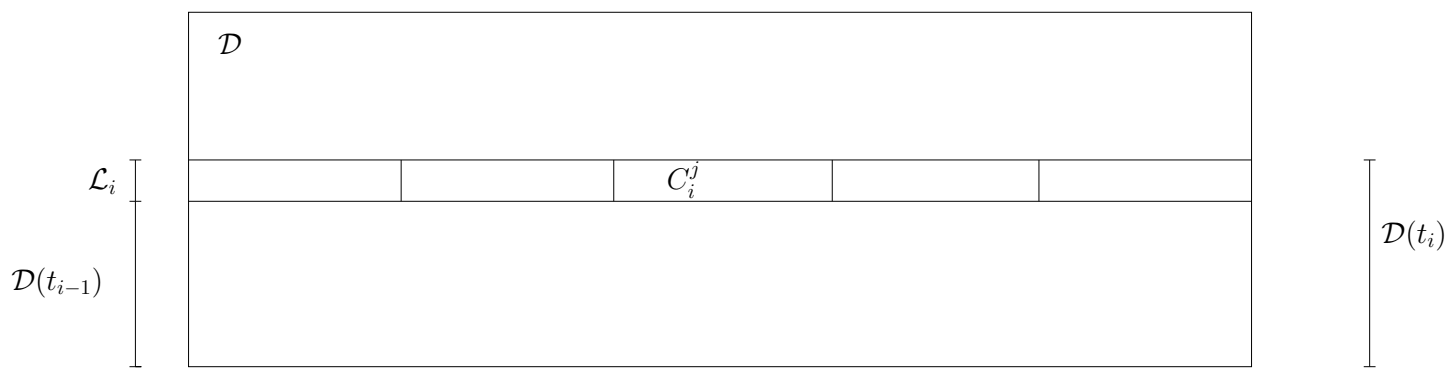

Figure 4: Intermediate structure $\mathcal{D}\left(t_{i}\right)$ with its last layer $\mathcal{L}_{i}$, made of macro-cells $C_{i}^{j}$, where $j$ is a time step index.

where $\tilde{T}_{i-1}$ is an extension of the previous temperature $T_{i-1}\left(t_{i-1}\right)$, defined as follows

$$
\tilde{T}_{i-1}= \begin{cases}T_{\text {init }} & \text { in } \mathcal{D}\left(t_{i}\right) \backslash \mathcal{D}\left(t_{i-1}\right), \\ T_{i-1}\left(t_{i-1}\right) & \text { in } \mathcal{D}\left(t_{i-1}\right),\end{cases}
$$

coupled with the thermoelastic system

$$
\begin{cases}-\operatorname{div}\left(\sigma_{i}\right)=f_{i} & \text { in }\left(t_{i-1}, t_{i}\right) \times \mathcal{D}\left(t_{i}\right), \\ \sigma_{i}=\sigma_{i}^{e l}+\sigma_{i}^{t h} & \text { in }\left(t_{i-1}, t_{i}\right) \times \mathcal{D}\left(t_{i}\right), \\ \sigma_{i}^{e l}=A e\left(u_{i}\right) & \sigma_{i}^{t h}=K\left(T_{i}-T_{i n i t}\right) \mathbb{I}_{n} \\ \sigma_{i} \cdot n=0 & \text { on }\left(t_{i-1}, t_{i}\right) \times \partial \mathcal{D}\left(t_{i}\right)_{N}, \\ u_{i}=0 & \text { on }\left(t_{i-1}, t_{i}\right) \times \partial \mathcal{D}\left(t_{i}\right)_{D}\end{cases}
$$

The equations of the model (24)-(25)-(26) are the same as those in the previous section, except that now the material properties and the domains vary with time. Note that, contrary to the previous section, there is no more a non-homogeneous Neumann boundary condition for the heat equation (24), since it is not necessary for the modelling of additive manufacturing. Therefore, for simplicity, the Fourier part of the boundary $\partial \mathcal{D}\left(t_{i}\right)_{F}$ for the heat equation is assumed to coincide with the homogeneous Neumann part of the boundary $\partial \mathcal{D}\left(t_{i}\right)_{N}$ for the thermoelastic system. As before, $f_{i}$ could be the gravity forces in each layer (and therefore would be independent of the index $i)$.

Note that problem (24) can be solved independently of (26), and that problem (26) depends on the temperature field $T_{i}$, solution of (24). Even with time dependent coefficients, it is well known [29] that (24) admits a unique solution $T_{i} \in C\left(\left[t_{i-1}, t_{i}\right] ; L^{2}\left(\mathcal{D}\left(t_{i}\right)\right)\right) \cap L^{2}\left(\left(t_{i-1}, t_{i}\right) ; H^{1}\left(\mathcal{D}\left(t_{i}\right)\right)\right)$. Extending $T_{i}$ outside $\mathcal{D}\left(t_{i}\right)$ by a formula similar to (25) allows us to define a global temperature fields $T$, equal to $T_{i}$ for $t_{i-1}<t<t_{i}$, which belongs to $C\left(\left[0, t_{f}\right] ; L^{2}(\mathcal{D})\right)$. Then, in turn $(26)$ admits a unique solution $u_{i} \in L^{2}\left(\left(t_{i-1}, t_{i}\right) ; H_{D}^{1}\left(\mathcal{D}\left(t_{i}\right)\right)^{n}\right)$.

\subsection{Optimization problem and shape derivative}

Before defining an optimization problem we recall that the shape $\Omega$ has to be optimized also with respect to its final utilization. We introduce the corresponding state equation which has completely different loads and boundary conditions with respect to the manufacturing model of the previous section. For some given surface loads $F$, we consider the following final utilization 
problem, which takes place after the layer by layer construction

$$
\begin{cases}-\operatorname{div}\left(A_{\Omega} e(u)\right)=0 & \text { in } \Omega \\ \left(A_{\Omega} e(u)\right) \cdot n=F & \text { on } \partial \Omega_{N} \\ \left(A_{\Omega} e(u)\right) \cdot n=0 & \text { on } \Gamma \\ u=0 & \text { on } \partial \Omega_{D}\end{cases}
$$

with $A_{\Omega}$, the constant elasticity tensor of the solid shape $\Omega, \partial \Omega=\partial \Omega_{N} \cup \partial \Omega_{D} \cup \Gamma$ where $\Gamma=\partial \Omega \backslash\left(\partial \Omega_{D} \cup \partial \Omega_{N}\right)$ only is free to move. Note that (27) is solved just in $\Omega$, without any more powder region, contrary to system (24)-(26) which is solved in the full subdomain $\mathcal{D}\left(t_{i}\right)$.

We consider an objective function which depends both on the final utilization of $\Omega$, as well as on its manufacturing process

$$
J(\Omega)=\int_{\partial \Omega_{N}} j_{1}(u) d x+\ell \sum_{i=1}^{M} \int_{t_{i-1}}^{t_{i}} \int_{\mathcal{D}\left(t_{i}\right)} j_{2}\left(u_{i}\right) d x d t,
$$

where $j_{1}$ and $j_{2}$ are two smooth functions with quadratic growth and $\ell$ is a fixed weighting coefficient. As mentioned before, (28) is just an example and many more choices are possible, for example depending on the gradient of $u_{i}$ or $u$ and on the temperature $T_{i}$, or involving a term at the final time. Our approach easily extends to such cases. Typical examples of functions $j_{1}, j_{2}$ are the compliance of the final structure and a least square penalization of the vertical displacement above a maximal threshold $u^{+}>0$ in the top layer $\mathcal{L}_{i}$ of each intermediate structure, during the building process,

$$
j_{1}(u)=F \cdot u \quad \text { and } \quad j_{2}(u)=\left|\max \left(u \cdot e_{d}-u^{+}, 0\right)\right|^{2} \chi_{\mathcal{L}_{i}} .
$$

The motivation for studying the objective function $j_{2}$ for the building process is that, if the intermediate structures are deformed too much in the vertical direction, it will prevent the rake or the roller to coat a new powder layer, therefore stopping the additive fabrication process.

Remark 3.3 Note that the variable in the objective function (28) is $\Omega$ while it was the interface $\Gamma$ in the previous section. This is just a matter of notations. The present notation encompasses the previous one since the interface between the solid and powder subdomains is a subset of the boundary $\partial \Omega$.

Associated to the objective function (28) we introduce the following adjoint systems.

- For the final displacement $u$

$$
\begin{cases}-\operatorname{div}\left(A_{\Omega} e(\eta)\right)=0 & \text { in } \Omega \\ \left(A_{\Omega} e(\eta)\right) \cdot n=0 & \text { on } \Gamma, \\ \left(A_{\Omega} e(\eta)\right) \cdot n=-j_{1}^{\prime}(u) & \text { on } \partial \Omega_{N} \\ \eta=0 & \text { on } \partial \Omega_{D}\end{cases}
$$

- For the intermediate displacements $u_{i}, 1 \leq i \leq M$,

$$
\begin{cases}-\operatorname{div}\left(A e\left(\eta_{i}\right)\right)=-j_{2}^{\prime}\left(u_{i}\right) & \text { in }\left(t_{i-1}, t_{i}\right) \times \mathcal{D}\left(t_{i}\right) \\ \left(A e\left(\eta_{i}\right)\right) \cdot n=0 & \text { on }\left(t_{i-1}, t_{i}\right) \times \partial \mathcal{D}\left(t_{i}\right)_{N}, \\ \eta_{i}=0 & \text { on }\left(t_{i-1}, t_{i}\right) \times \partial \mathcal{D}\left(t_{i}\right)_{D} .\end{cases}
$$


- For the intermediate temperatures $T_{i}, 1 \leq i \leq M$,

$$
\begin{cases}\rho \frac{\partial p_{i}}{\partial t}+\operatorname{div}\left(\lambda \nabla p_{i}\right)=K \operatorname{div}\left(\eta_{i}\right) & \text { in }\left(t_{i-1}, t_{i}\right) \times \mathcal{D}\left(t_{i}\right), \\ \left(\lambda \nabla p_{i}\right) \cdot n=-\beta p_{i} & \text { on }\left(t_{i-1}, t_{i}\right) \times \partial \mathcal{D}\left(t_{i}\right)_{N}, \\ p_{i}=0 & \text { on }\left(t_{i-1}, t_{i}\right) \times \partial \mathcal{D}\left(t_{i}\right)_{D}, \\ p_{i}\left(t_{i}\right)=p_{i+1}\left(t_{i}\right) & \text { in } \mathcal{D}\left(t_{i}\right),\end{cases}
$$

with the convention that, for the last layer $i=M$, the final condition is

$$
p_{M+1}\left(t_{M}\right)=0 \text { in } \mathcal{D}\left(t_{M}\right) \text {. }
$$

Theorem 3.1 Let $\theta \in W^{1, \infty}(\mathcal{D}, \mathcal{D})$ be such that $\theta \cdot n=0$ on $\partial \mathcal{D}$ and $\theta=0$ on $\partial \Omega_{D} \cup \partial \Omega_{N}$. Under Assumption 2.2 the shape derivative of $J(\Omega)$ is

$$
\begin{aligned}
J^{\prime}(\Omega)(\theta) & =\ell \sum_{i=1}^{M} \int_{t_{i-1}}^{t_{i}} \int_{\Gamma \cap \mathcal{D}\left(t_{i}\right)} \theta \cdot n\left\{\left(\rho_{\Omega}-\rho_{P}\right) \frac{\partial T_{i}}{\partial t} p_{i}+\left(\lambda_{\Omega}-\lambda_{P}\right) \nabla T_{i} \cdot \nabla p_{i}-\left(Q_{\Omega}^{i}-Q_{P}^{i}\right) p_{i}\right\} d s d t \\
& +\ell \sum_{i=1}^{M} \int_{t_{i-1}}^{t_{i}} \int_{\Gamma \cap \mathcal{D}\left(t_{i}\right)} \theta \cdot n\left\{\left(\left(A_{\Omega}-A_{P}\right) e\left(u_{i}\right)+\left(K_{\Omega}-K_{P}\right)\left(T_{i}-T_{i n i t}\right) \mathbb{I}_{n}\right): e\left(\eta_{i}\right)-\left(f_{\Omega}^{i}-f_{P}^{i}\right) \cdot \eta_{i}\right\} d s d t \\
& +\ell \sum_{i=1}^{M} \int_{t_{i-1}}^{t_{i}} \int_{\partial \mathcal{D}\left(t_{i}\right)_{N} \cap \partial \Gamma}\left(\beta_{\Omega}-\beta_{P}\right)\left(T_{i}-T_{i n i t}\right) p_{i} \theta \cdot \tau d L d t \\
& +\int_{\Gamma} \theta \cdot n\left(A_{\Omega} e(u): e(\eta)\right) d s,
\end{aligned}
$$

where $\eta, \eta_{1}, . ., \eta_{M}, p_{1}, . ., p_{M}$, are the (discrete in space) solutions of the adjoint problems (29), (30), (31) and (32), $n$ is the normal unit vector to $\partial \Omega$ (oriented from $\Omega$ to $\mathcal{D}\left(t_{i}\right) \backslash \Omega$ ), and $\tau$ is the unit vector tangent to $\partial \Omega$ and normal to $\partial\left(\partial \mathcal{D}\left(t_{i}\right) \cap \partial \Omega\right)$.

Proof The idea of the proof follows again the classical Lagrangian method of Céa. One introduces the Lagrangian functional

$$
\begin{aligned}
& \mathcal{L}\left(\Gamma, \hat{u}, \hat{\eta}, \hat{u}_{1}, \ldots, \hat{u}_{M}, \hat{\eta}_{1}, \ldots, \hat{\eta}_{M}, \hat{T}_{1}, \ldots, \hat{T}_{M}, \hat{p}_{1}, \ldots, \hat{p}_{M}\right)= \\
& =\int_{\partial \Omega_{N}} j_{1}(\hat{u}) d x+\ell \sum_{i=1}^{M} \int_{t_{i-1}}^{t_{i}} \int_{\mathcal{D}\left(t_{i}\right)} j_{2}\left(\hat{u}_{i}\right) d x d t+\sum_{i=1}^{M} \int_{t_{i-1}}^{t_{i}} \int_{\mathcal{D}\left(t_{i}\right)}\left\{\rho \frac{\partial \hat{T}_{i}}{\partial t} \hat{p}_{i}+\lambda \nabla \hat{T}_{i} \cdot \nabla \hat{p}_{i}-Q \hat{p}_{i}\right\} d x d t \\
& +\sum_{i=1}^{M} \int_{t_{i-1}}^{t_{i}} \int_{\partial \mathcal{D}_{N}\left(t_{i}\right)} \beta\left(\hat{T}_{i}-T_{i n i t}\right) \hat{p}_{i} d s d t-\sum_{i=1}^{M} \int_{t_{i-1}}^{t_{i}} \int_{\partial \mathcal{D}_{D}\left(t_{i}\right)}\left\{\left(\lambda \nabla \hat{T}_{i}\right) \cdot n \hat{p}_{i}+\left(\lambda \nabla \hat{p}_{i}\right) \cdot n\left(\hat{T}_{i}-T_{i n i t}\right)\right\} d s d t \\
& +\sum_{i=1}^{M} \int_{\mathcal{D}\left(t_{i}\right)}\left(\hat{T}_{i}\left(t_{i-1}\right)-\hat{\tilde{T}}_{i-1}\right) \rho \hat{p}_{i}\left(t_{i-1}\right) d x+\sum_{i=1}^{M} \int_{t_{i-1}}^{t_{i}} \int_{\mathcal{D}\left(t_{i}\right)}\left\{\left(A e\left(\hat{u}_{i}\right)+K\left(\hat{T}_{i}-T_{i n i t}\right) \mathbb{I}_{n}\right): e\left(\hat{\eta}_{i}\right)-f_{i} \cdot \hat{\eta}_{i}\right\} d x d t \\
& +\int_{\Omega} A_{\Omega} e(\hat{u}): e(\hat{\eta}) d x-\int_{\partial \Omega_{N}} F \cdot \hat{\eta} d s,
\end{aligned}
$$

where $\hat{u}, \hat{\eta} \in V_{h 0}^{n}, \hat{u}_{i}, \hat{\eta}_{i}, \in L^{2}\left(\left[t_{i-1}, t_{i}\right], V_{h 0}^{n}\right)$ and $\hat{T}_{i}, \hat{p}_{i} \in C\left(\left[t_{i-1}, t_{i}\right], V_{h}\right)$. Differentiating $\mathcal{L}$ with respect to each of the previous variables and following the proof of Theorem 2.4 the desired result is easily obtained. 


\subsection{Shape derivative for a discrete time additive process}

We now establish a discrete time (index $j$ ) shape derivative result that take account the layers (index $i$ ). We discretize each time interval $\left(t_{i-1}, t_{i}\right)$ in $N$ subintervals $\left(t_{i}^{j-1}, t_{i}^{j}\right)$ of the length $\Delta t=t_{i}^{j}-t_{i}^{j-1}=\left(t_{i}-t_{i-1}\right) / N$ such that

$$
t_{i-1}=t_{i}^{0}<t_{i}^{1}<\ldots<t_{i}^{N-1}<t_{i}^{N}=t_{i} .
$$

Recall that, between times $t_{i-1}$ and $t_{i}$, only the last layer $\mathcal{L}_{i}$ in domain $\mathcal{D}\left(t_{i}\right)$ has changing material properties. Thus, for $t_{i-1}<t_{i}^{j}<t_{i}$, we define a discrete version of the material coefficient

$$
\xi_{i}^{j}:=\xi\left(t_{i}^{j}, x\right)= \begin{cases}\xi_{\Omega} \chi_{\Omega}(x)+\xi_{\mathcal{P}} \chi_{\mathcal{P}}(x) & \text { if } x \in \mathcal{D}\left(t_{i-1}\right), \\ \xi_{\Omega} \chi_{\mathcal{L}}\left(t_{i}^{j}, x\right)+\xi_{\mathcal{P}}\left(1-\chi_{\mathcal{L}}\left(t_{i}^{j}, x\right)\right) & \text { if } x \in \mathcal{L}_{i} .\end{cases}
$$

For $1 \leq j \leq N$, we define on the subinterval $\left(t_{i}^{j-1}, t_{i}^{j}\right)$ the discrete time evolution $\left(u_{i}^{j}, T_{i}^{j}\right)$ as a solution of the following system of the equations.

- Heat equation:

$$
\begin{cases}\rho_{i}^{j} \frac{T_{i}^{j}-T_{i}^{j-1}}{\Delta t}-\operatorname{div}\left(\lambda_{i}^{j} \nabla T_{i}^{j}\right)=Q_{i}^{j} & \text { in } \mathcal{D}\left(t_{i}\right) \\ \left(\lambda_{i}^{j} \nabla T_{i}^{j}\right) \cdot n=-\beta_{i}^{j}\left(T_{i}^{j}-T_{\text {init }}\right) & \text { on } \partial \mathcal{D}\left(t_{i}\right)_{N}, \\ T_{i}^{j}=T_{\text {init }} & \text { on } \partial \mathcal{D}\left(t_{i}\right)_{D}\end{cases}
$$

with the initial condition

$$
T_{i}^{j=0}=\tilde{T}_{i-1}= \begin{cases}T_{i n i t} & \text { in } \mathcal{D}\left(t_{i}\right) \backslash \mathcal{D}\left(t_{i-1}\right), \\ T_{i-1}^{N} & \text { in } \mathcal{D}\left(t_{i-1}\right)\end{cases}
$$

Remark 3.4 The initial condition $T_{i}^{j=0}$ for the temperature field in the domain $\mathcal{D}\left(t_{i}\right)$ is imposed as follows. In the previous domain $\mathcal{D}\left(t_{i-1}\right)$, the initial temperature $T_{i}^{j=0}$ at the first time step $j=0$ is taken equal to the temperature at the last time step $T_{i-1}^{N}$, namely $T_{i}^{j=0}=T_{i-1}^{N}$ in $\mathcal{D}\left(t_{i-1}\right)$. However, in $\mathcal{D}\left(t_{i}\right) \backslash \mathcal{D}\left(t_{i-1}\right)$, which was not part of the previous computational domain, the temperature $T_{i}^{j=0}$ is chosen equal to $T_{\text {init }}$.

- Thermoelastic equilibrium system:

$$
\begin{cases}-\operatorname{div}\left(\sigma_{i}^{j}\right)=f_{i}^{j} & \text { in } \mathcal{D}\left(t_{i}\right) \\ \sigma_{i}^{j}=\sigma_{j}^{e l}+\sigma_{j}^{t h} & \text { in } \mathcal{D}\left(t_{i}\right) \\ \sigma_{i e l}^{j}=A_{i}^{j} e\left(u_{i}^{j}\right) & \sigma_{i t h}^{j}:=K_{i}^{j}\left(T_{i}^{j}-T_{i n i t}\right) \mathbb{I}_{n} \\ \sigma_{i}^{j} \cdot n=0 & \text { on } \partial \mathcal{D}\left(t_{i}\right)_{N} \\ u_{i}^{j}=0 & \text { on } \partial \mathcal{D}\left(t_{i}\right)_{D}\end{cases}
$$

where $f_{i}^{j}$ is an approximation of $f_{i}(t)$ at time $t=t_{i}^{j}$.

We consider the following (time discrete) objective function

$$
J_{\Delta t}(\Omega)=\int_{\partial \Omega_{N}} j_{1}(u) d x+\ell \sum_{i=1}^{M} \Delta t \sum_{j=1}^{N} \int_{\mathcal{D}\left(t_{i}\right)} j_{2}\left(u_{i}^{j}\right) d x .
$$

Associated to this objective function (38) we introduce the following adjoint systems. 
- For the final displacement $u$ (which is independent of time), the adjoint equation is still (29).

- For the intermediate displacements $u_{i}^{j}$

$$
\begin{cases}-\operatorname{div}\left(A_{i}^{j} e\left(\eta_{i}^{j}\right)\right)=-j_{2}^{\prime}\left(u_{i}^{j}\right) & \text { in } \mathcal{D}\left(t_{i}\right) \\ \left(A_{i}^{j} e\left(\eta_{i}^{j}\right)\right) \cdot n=0 & \text { on } \partial \mathcal{D}\left(t_{i}\right)_{N} \\ \eta_{i}^{j}=0 & \text { on } \partial \mathcal{D}\left(t_{i}\right)_{D}\end{cases}
$$

- For the intermediate temperatures $T_{i}^{j}$, for all layers $1 \leq i \leq M$ and for all time steps $1 \leq j \leq N$

$$
\begin{cases}\rho_{i}^{j} \frac{p_{i}^{j+1}-p_{i}^{j}}{\Delta t}+\operatorname{div}\left(\lambda_{i}^{j} \nabla p_{i}^{j}\right)=K_{i}^{j} \operatorname{div}\left(\eta_{i}^{j}\right) & \text { in } \mathcal{D}\left(t_{i}\right), \\ \left(\lambda_{i}^{j} \nabla p_{i}^{j}\right) \cdot n=-\beta_{i}^{j} p_{i}^{j} & \text { on } \partial \mathcal{D}\left(t_{i}\right)_{N}, \\ p_{i}^{j}=0 & \text { on } \partial \mathcal{D}\left(t_{i}\right)_{D},\end{cases}
$$

with the final condition at time $t_{i}^{N}$ for $i<M$

$$
p_{i}^{N+1}=p_{i+1}^{1}
$$

and at time $t_{M}^{N}$

$$
p_{M}^{N+1}=0 .
$$

As usual, the adjoint systems (40) have to be solved backward in time. More precisely, one decreases the indices $i$ from $M$ to 1 and $j$ from $N$ to 1 . The ultimate final condition is (42) but the final condition of each intermediate layer is (41) which combines the final time step of layer $i$ with the initial time step of layer $i+1$. Note that $p_{i+1}^{1}$ is defined in a domain larger than $p_{i}^{N+1}$, so only its restriction to $\mathcal{D}\left(t_{i}\right)$ is required.

Theorem 3.2 Let $\theta \in W^{1, \infty}(\mathcal{D}, \mathcal{D})$ be such that $\theta \cdot n=0$ on $\partial \mathcal{D}$ and $\theta=0$ on $\partial \Omega_{D} \cup \partial \Omega_{N}$. Under Assumption 2.2 the shape derivative of $J(\Omega)$ is

$$
\begin{aligned}
J_{\Delta t}^{\prime}(\Omega)(\theta) & =\ell \sum_{i=1}^{M} \Delta t \sum_{j=1}^{N} \int_{\Gamma \cap \mathcal{D}\left(t_{i}\right)} \theta \cdot n\left\{\left(\rho_{\Omega}-\rho_{P}\right) \frac{T_{i}^{j}-T_{i}^{j-1}}{\Delta t} p_{i}^{j}+\left(\lambda_{\Omega}-\lambda_{P}\right) \nabla T_{i}^{j} \cdot \nabla p_{i}^{j}-\left(Q_{i \Omega}^{j}-Q_{i P}^{j}\right) p_{i}^{j}\right\} d s \\
& +\ell \sum_{i=1}^{M} \Delta t \sum_{j=1}^{N} \int_{\Gamma \cap \mathcal{D}\left(t_{i}\right)} \theta \cdot n\left\{\left(\left(A_{\Omega}-A_{P}\right) e\left(u_{i}^{j}\right)+\left(K_{\Omega}-K_{P}\right)\left(T_{i}^{j}-T_{i n i t}\right) \mathbb{I}_{n}\right): e\left(\eta_{i}^{j}\right)-\left(f_{i \Omega}^{j}-f_{i P}^{j}\right) \cdot \eta_{i}^{j}\right\} \\
& +\ell \sum_{i=1}^{M} \Delta t \sum_{j=1}^{N} \int_{\partial \mathcal{D}\left(t_{i}\right)_{N} \cap \partial \Gamma}\left(\beta_{\Omega}-\beta_{P}\right)\left(T_{i}^{j}-T_{i n i t}\right) p_{i}^{j} \theta \cdot \tau d L \\
& +\int_{\Gamma} \theta \cdot n\left(A_{\Omega} e(u): e(\eta)\right) d s
\end{aligned}
$$

where $\eta, \eta_{i}^{j}, p_{i}^{j}$, are the (discrete in space) solutions of the adjoint problems (29), (39), (40), (41), (42), $n$ is the normal unit vector to $\partial \Omega$ (oriented from $\Omega$ to $\mathcal{D}\left(t_{i}\right) \backslash \Omega$ ), and $\tau$ is the unit vector tangent to $\partial \Omega$ and normal to $\partial\left(\partial \mathcal{D}\left(t_{i}\right) \cap \partial \Omega\right)$. 
Proof The proof is very similar to that of Theorem 3.1. Thus, we content ourselves in giving the definition of the necessary Lagrangian

$$
\begin{aligned}
& \mathcal{L}\left(\Gamma, \hat{u}, \hat{\eta}, \hat{u}_{i}^{j}, \hat{\eta}_{i}^{j}, \hat{T}_{i}^{j}, \hat{p}_{i}^{j}\right)= \\
= & \int_{\partial \Omega_{N}} j_{1}(\hat{u}) d x+\int_{\Omega} A_{\Omega} e(\hat{u}): e(\hat{\eta}) d x-\int_{\partial \Omega_{N}} F \cdot \hat{\eta} d s \\
+ & \ell \sum_{i=1}^{M} \Delta t \sum_{j=1}^{N} \int_{\mathcal{D}\left(t_{i}\right)} j_{2}\left(\hat{u}_{i}^{j}\right) d x+\sum_{i=1}^{M} \Delta t \sum_{j=1}^{N} \int_{\mathcal{D}\left(t_{i}\right)}\left\{\rho_{i}^{j} \frac{\hat{T}_{i}^{j}-\hat{T}_{i}^{j-1}}{\Delta t} \hat{p}_{i}^{j}+\lambda_{i}^{j} \nabla \hat{T}_{i}^{j} \cdot \nabla \hat{p}_{i}^{j}-Q_{i}^{j} \hat{p}_{i}^{j}\right\} d x \\
+ & \sum_{i=1}^{M} \Delta t \sum_{j=1}^{N} \int_{\partial \mathcal{D}_{N}\left(t_{i}\right)} \beta_{i}^{j}\left(\hat{T}_{i}^{j}-T_{i n i t}\right) \hat{p}_{i}^{j} d s-\sum_{i=1}^{M} \Delta t \sum_{j=1}^{N} \int_{\partial \mathcal{D}_{D}\left(t_{i}\right)}\left\{\left(\lambda_{i}^{j} \nabla \hat{T}_{i}^{j}\right) \cdot n \hat{p}_{i}^{j}+\left(\lambda_{i}^{j} \nabla \hat{p}_{i}^{j}\right) \cdot n\left(\hat{T}_{i}^{j}-T_{i n i t}\right)\right\} d s \\
+ & \sum_{i=1}^{M} \int_{\mathcal{D}\left(t_{i}\right)}\left(\hat{T}_{i}^{0}-\hat{\tilde{T}}_{i-1}\right) \rho_{i}^{1} \hat{p}_{i}^{1} d x+\sum_{i=1}^{M} \Delta t \sum_{j=1}^{N} \int_{\mathcal{D}\left(t_{i}\right)}\left\{\left(A_{i}^{j} e\left(\hat{u}_{i}^{j}\right)+K_{i}^{j}\left(\hat{T}_{i}^{j}-T_{i n i t}\right) \mathbb{I}_{n}\right): e\left(\hat{\eta}_{i}^{j}\right)-f_{i}^{j} \cdot \hat{\eta}_{i}^{j}\right\} d x
\end{aligned}
$$

where $\hat{u}, \hat{\eta} \in V_{h 0}^{n}, \hat{u}_{i}^{j}, \hat{\eta}_{i}^{j}, \in V_{h 0}^{n}$ and $\hat{T}_{i}^{j}, \hat{p}_{i}^{j} \in V_{h}$.

\section{Computational algorithm}

\subsection{From shape derivative to velocity in Hamilton-Jacobi equation}

This section recalls the basic numerical ingredients for our computations, following the approach initiated in [6]. In other words, it explains how the shape derivative of the objective function $J(\Omega)$ is used in numerical practice. (As explained in Remark 3.3 the notation $J(\Gamma)$ is sometimes used instead of $J(\Omega)$, where $\Gamma \subset \partial \Omega$ is the interface between the solid and powder subdomains ; it is just a matter of notations and does not change anything to the present section.) Let $\mathcal{D} \subset \mathbb{R}^{n}$ be a bounded working domain in which all admissible shapes $\Omega$ are included ( $\mathcal{D}$ will be later uniformly meshed). In order to minimize the objective function, the shape $\Omega$ is deformed in a descent direction $\theta \in W^{1, \infty}(\mathcal{D}, \mathcal{D})$ such that the directional derivative $J^{\prime}(\Omega)(\theta)$ is negative. Recall that the general form of a shape derivative is

$$
J^{\prime}(\Omega)(\theta)=\int_{\partial \Omega} v \theta \cdot n d s
$$

where $v(x)$ is some integrand function, depending on the choice of $J(\Omega)$ and given by a result like Theorem 2.5. Therefore a possible descent direction is simply given by $\theta=-v n$. Introducing a small descent step $t>0$, the shape $\Omega$ is updated as $\Omega_{t}=(\operatorname{Id}+t \theta) \Omega$. Formally, it holds

$$
J\left(\Omega_{t}\right)=J(\Omega)-t \int_{\partial \Omega} v^{2} d s+\mathcal{O}\left(t^{2}\right),
$$

and thus, for small enough $t>0$, the objective function is decreased if $v \neq 0$. However, for numerical reasons (namely in order to regularize the function $v$ and to extend it from $\partial \Omega$ to the whole domain $\mathcal{D}$ ), following [6], we choose another descent direction $\theta=\mathcal{V} n$ where $\mathcal{V}$ is the unique solution in $H^{1}(\mathcal{D})$ of the variational formulation

$$
\int_{\mathcal{D}}\left(\epsilon^{2} \nabla \mathcal{V} \cdot \nabla \phi+\mathcal{V} \phi\right) d x=-\int_{\partial \Omega} v \phi d s \quad \text { for all } \phi \in H^{1}(\mathcal{D}),
$$


where $\epsilon>0$ is a small parameter (typically of the order of the mesh size). One can easily check from (43) that, indeed, $\theta=\mathcal{V} n$ is a descent direction, i.e., $J^{\prime}(\Omega)(\mathcal{V} n) \leq 0$. In practice, since the boundary $\partial \Omega$ is not exactly meshed, the right hand side boundary integral of (43) is replaced by

$$
-\int_{\mathcal{D}} \delta_{\partial \Omega} v \phi d x
$$

where $\delta_{\partial \Omega}$ is a numerical approximation of the Dirac mass function carried by the boundary $\partial \Omega$ (see [6], [7] for details).

\subsection{Hamilton-Jacobi equation for level set optimization}

The deformation or update of the shape $\Omega$ in $\Omega_{t}=(\operatorname{Id}+t \theta) \Omega$, for $t \geq 0$ and $\theta=\mathcal{V} n$, is performed by means of the level set method of Osher and Sethian [32]. The initial shape $\Omega$ is implicitly represented on the working domain $\mathcal{D}$ by a so-called level set function $\psi_{0}$, satisfying

$$
\begin{cases}\psi_{0}(x)=0 & \text { iff } x \in \partial \Omega \cap \mathcal{D} \\ \psi_{0}(x)<0 & \text { iff } x \in \Omega \\ \psi_{0}(x)>0 & \text { iff } x \in \mathcal{D} \backslash \bar{\Omega} .\end{cases}
$$

Numerically, the discretization of the shape $\Omega$ is captured by the discrete values of $\psi_{0}$ on the fixed mesh of $\mathcal{D}$.

Moving $\Omega$ to $\Omega_{t}=(\operatorname{Id}+t \mathcal{V} n) \Omega$ is equivalent to solve, from the initial time 0 to the final time $t$, the following Hamilton-Jacobi equation

$$
\begin{cases}\frac{\partial \psi}{\partial t}+\mathcal{V}|\nabla \psi|=0 & \text { in }(0, t) \times \mathcal{D}, \\ \psi(0, x)=\psi_{0}(x) & \text { in } \mathcal{D}\end{cases}
$$

where the normal velocity $\mathcal{V}$ is the solution of (43). Of course, the time variable $t$ in the above eikonal equation is not the true time variable (as in the models of Sections 2 and 3 ) but is a pseudo-time, corresponding to the descent step in optimization. The shape $\Omega_{t}$ is simply recovered as the set of points $x \in \mathcal{D}$ such that $\psi(t, x)<0$. Eventually, if constraints on $\Omega$ have to be taken into account in the optimization (for example, weight or perimeter constraints), there are aggregated to the objective function, via Lagrange multipliers, into a Lagrangian to which the above approach is applied. More precisely, our optimization algorithm is an augmented Lagrangian method which is able to take into account both equality and inequality constraints.

\subsection{Algorithm}

In summary our algorithm for the model of Section 2 is as follows (it is easily extended to the model of Section 3).

1. Initialization : $\psi_{0}$

2. Iteration until convergence : for $k \geq 0$

- Computation of the discrete time solutions $u_{k}^{j}, T_{k}^{j}, \eta_{k}^{j}, p_{k}^{j}$, for the successive time increments $1 \leq j \leq N$, with the current shape $\psi_{k}$.

- Computation of the shape derivative and the normal velocity $\mathcal{V}_{k}$ via the variational problem (43). 
- Computation of $\psi_{k+1}$, solution of the Hamilton-Jacobi equation (44) with normal velocity $\mathcal{V}_{k}$ and initial condition $\psi_{k}$. The final time (or descent step) $t_{k}>0$ in (44) is choosen such that $J\left(\psi_{k+1}\right) \leq J\left(\psi_{k}\right)$.

- If the norm of $\mathcal{V}_{k}$ is small enough, or if $t_{k}$ becomes too small, then convergence is detected and iterations are stopped.

3. From time to time, the level set function $\psi_{k}$ is reinitialized as the signed distance function to the current shape $\Omega_{k}$ (using the algorithm of [14]).

\subsection{Regularization of the interface between two phases}

We recall that the domain $\mathcal{D}$ is decomposed into two sub-domains $\Omega_{1}$ and $\Omega_{2}$ (as in Section 2), and that each intermediate domain $\mathcal{D}\left(t_{i}\right)$, after completion at time $t_{i}$ (as in Section 3) was decomposed into two sub-domains $\Omega\left(t_{i}\right)$ and $\mathcal{P}\left(t_{i}\right)$, occupied by two different materials, solid and powder, respectively. In other words, $\Omega\left(t_{i}\right)$ played the role of $\Omega_{1}$ and $\mathcal{P}\left(t_{i}\right)$ that of $\Omega_{2}$. In numerical practice the interface $\Gamma$ between $\Omega_{1}$ and $\Omega_{2}$ is not exactly meshed. Still, it could be a sharp interface, meaning that the transition between the subdomains $\Omega_{1}$ and $\Omega_{2}$ takes place in a single layer of mesh cells. However, as explained in [1], for numerical efficiency, it is better to model the interface $\Gamma$ as a thick interface of (small) width $2 \varepsilon>0$ where $\varepsilon$ is of the order of a few mesh-cell size. Of course, the interface should not diffuse, that is, get enlarged during the optimization process. Therefore, the interface thickness is kept fixed thanks to another ingredient linked to the level set method, namely the signed distance function to the sharp interface. As proved in [1], the following definition guarantees that the thick numerical interface will never get larger than the prescribed value $2 \varepsilon$.

More precisely, given two material parameterss $\xi_{1}$ and $\xi_{2}$, corresponding respectively to the subdomains $\Omega_{1}$ and $\Omega_{2}$, we replace the sharp interface definition (1) of their mixture by the following one

$$
\xi_{\varepsilon}(x)=\xi_{1}+h_{\varepsilon}\left(d_{\Omega_{1}}(x)\right)\left(\xi_{2}-\xi_{1}\right),
$$

where, following the approach in [1], $d_{\Omega_{1}}(x)$ is the signed distance function to $\Omega_{1}$ and, for $\varepsilon>0$, $h_{\varepsilon}$ is a smooth approximation of the Heavside's function

$$
h_{\varepsilon}(z)= \begin{cases}0 & \text { if } z<-\varepsilon \\ \frac{1}{2}\left(1+\frac{z}{\varepsilon}+\frac{1}{\pi} \sin \left(\frac{\pi z}{\varepsilon}\right)\right) & \text { if }-\varepsilon \leq z \leq \varepsilon, \\ 1 & \text { if } z>\varepsilon\end{cases}
$$

\section{$5 \quad$ Numerical simulations}

\subsection{Setting of the test cases}

In this section we present several numerical results for the shape optimization problems concerning the minimization of the thermal stresses and of the vertical displacement (to allow the roller to coat a new powder layer). The related objective functions are:

- for the deviatoric part of the stress $\sigma_{D}=\left(A e(u)+K\left(T-T_{\text {init }}\right) \mathbb{I}_{n}\right)_{D}=2 \mu e(u)_{D}$

$$
J_{1}(\Omega)=\sum_{i=1}^{M} \int_{t_{i-1}}^{t_{i}} \int_{\mathcal{D}\left(t_{i}\right)}\left|\sigma_{D}\right|^{2} d x d t
$$

where the deviatoric part of a $n \times n$ square matrix $\xi$ is $\xi_{D}=\xi-\frac{\operatorname{tr}(\xi)}{n} \mathbb{I}_{n}$, 
- for the vertical displacement

$$
J_{2}(\Omega)=\sum_{i=1}^{M} \int_{t_{i-1}}^{t_{i}} \int_{\mathcal{D}\left(t_{i}\right) \backslash \mathcal{D}\left(t_{i-1}\right)}\left|\max \left(0, u \cdot e_{d}-u^{+}\right)\right|^{2} d x d t,
$$

where $u^{+}>0$ is a desired maximal vertical displacement.

We define the following quantities

- the weight (or volume)

$$
|\Omega|=\int_{\mathcal{D}}\left(1-h_{\varepsilon}\left(d_{\Omega}(x)\right)\right) d x,
$$

- the compliance

$$
C(\Omega)=\int_{\partial \Omega_{N}} F \cdot u_{\Omega} d s,
$$

where $u_{\Omega}$ is the solution of problem (27) for the final use of the shape $\Omega$.

All our test cases will start from an initialization $\Omega_{r e f}$ which is a reference shape, obtained by compliance minimization for its final use (that is, without any manufacturing constraint). Therefore, for one of the two above objective functions, $J_{1}$ or $J_{2}$, we consider the following minimization problem

$$
\begin{aligned}
\min _{\Omega \subset \mathcal{D}} & J_{i}(\Omega) \\
\text { s.t. } & |\Omega|=\left|\Omega_{r e f}\right| \\
& C(\Omega) \leq(1+\kappa) C\left(\Omega_{r e f}\right),
\end{aligned}
$$

where $\kappa>0$ is a small parameter, allowing for a slight increase of the compliance of the final structure (typically $\kappa=0.05$ or 0.1 ). Note that we enforce the same weight for the admissible shapes $\Omega$ than for the reference and initial shape $\Omega_{r e f}$. Our optimization algorithm is an augmented Lagrangian method which is able to take into account both equality (weight) and inequality (compliance) constraints.

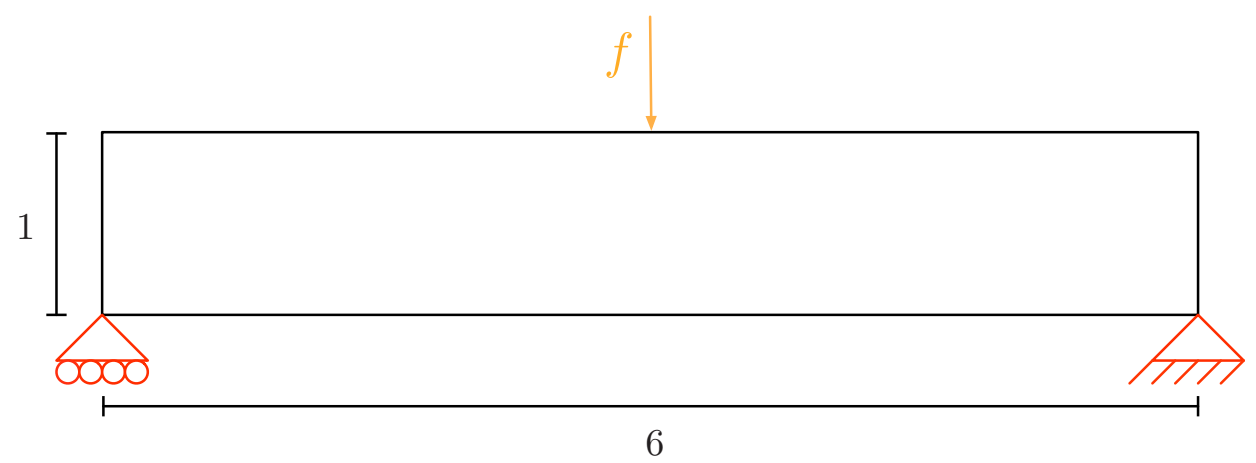

Figure 5: Boundary conditions and loading for the two-dimensional MBB beam.

All our test cases are concerned with the MBB beam (in 2-d as well as in 3-d). The loading and boundary conditions in 2-d for (27) are displayed on Figure 5. In 3-d the applied load is 


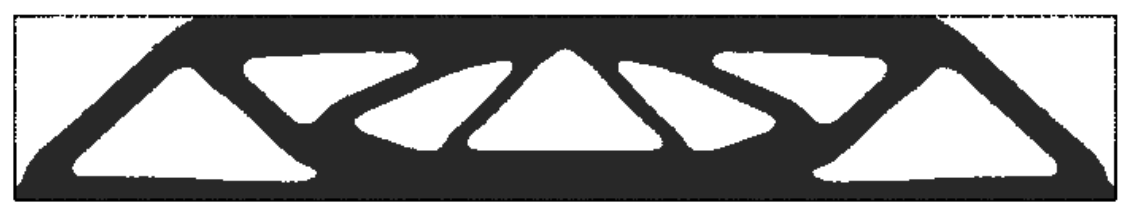

Figure 6: Optimal MBB beam for compliance minimization which is the initialization and reference shape for the constrained optimization (48)

similarly applied at the middle of the top face of the beam and the Dirichlet boundary conditions are applied at the four lower corners. By symmetry the computation is performed on half the domain. The computational domain is thus $3 \mathrm{~cm} \times 1 \mathrm{~cm} \times 1 \mathrm{~cm}$ in 3 -d and $3 \mathrm{~cm} \times 1 \mathrm{~cm}$ in 2 -d. The load is $F=(0,-0.5)$ in 2 -d, applied on a boundary part of length $0.05 \mathrm{~cm}$ and $F=(0,0,-1)$ in $3-\mathrm{d}$, applied on a boundary part of area $0.05 \mathrm{~cm}^{2}$.

We use the finite element software FreeFem $++[22]$. In 2-d the triangular mesh has 5359 nodes and in 3-d 20538 nodes. The reference shape $\Omega_{r e f}$ for the MBB beam is obtained by compliance minimization and can be seen on Figure 6 in 2-d (with a volume of $52 \%$ of the computational domain) and on Figure 29 (left column) in 3-d (with a volume of $38 \%$ of the computational domain).

In all the following numerical calculations, material 1 denotes the solid (typically a maraging steel) and material 2 is the metallic powder. Our mechanical parameters are taken from [41]. Their values with physical units are: $E_{1}=200 \mathrm{GPa}, \nu_{1}=\nu_{2}=0.3$, the heat capacity $C_{1}=$ $C_{2}=450 \mathrm{~J} . \mathrm{kg}^{-1} \cdot{ }^{\circ} \mathrm{C}^{-1}$, the heat transfer coefficient $\beta_{1}=\beta_{2}=10 \mathrm{~W} \cdot \mathrm{m}^{-2} .{ }^{\circ} \mathrm{C}^{-1}, \alpha_{1}=0.000015$ ${ }^{\circ} C^{-1}, \tilde{\rho}_{1}=8000 \mathrm{~kg} . \mathrm{m}^{-3}, \lambda_{1}=15 \mathrm{~W} \cdot \mathrm{m}^{-1} \cdot{ }^{\circ} \mathrm{C}^{-1}, \alpha_{2}=0.000001{ }^{\circ} \mathrm{C}^{-1}, E_{2}=1.6 \mathrm{GPa}, \tilde{\rho}_{2}=4000$ $\mathrm{kg} \cdot \mathrm{m}^{-3}, \lambda_{2}=0.25 \mathrm{~W} \cdot \mathrm{m}^{-1} \cdot{ }^{\circ} \mathrm{C}^{-1}$. Remark that, for simplicity, our notation in the heat equation is $\rho_{1}=C_{1} \tilde{\rho}_{1}$ and $\rho_{2}=C_{2} \tilde{\rho}_{2}$.

For each test case, we shall specify the following parameters: the time discretization step $\Delta t$, the number $N$ of time steps for the model of section 2 or of time steps in each layer for the model of Section 3, the number of layers $M$, the heat fluxes applied in the solid $Q_{1}$ and in the powder $Q_{2}$, the thickness regularization parameter $\varepsilon$ and the regularization parameter $\epsilon$ introduced in Section 4.1.

\subsection{Simplified model of Section 2 in 2 -d}

We first consider the simplified model of Section 2 which does not feature the layer by layer construction and applies a constant heat source term to the final shape $\Omega$. Such a model permits a faster computation than the full model of Section 3. Recall that $\Omega$ plays the role of $\Omega_{1}$ and $\mathcal{D} \backslash \Omega$ that of $\Omega_{2}$. The numerical parameters are: $t_{f}=1, \Delta t=0.1 \mathrm{~s}, N=10, Q_{1}=8000 \mathrm{~W} . \mathrm{cm}^{-2}$ (in 2-d), $Q_{2}=10^{-3} Q_{1}, \varepsilon=2 \Delta x, \epsilon=\varepsilon$ with a mesh size of the order of $\Delta x=0.04$. The value of the source term $Q_{1}$ does not correspond to the laser power since it is applied in the full final shape $\Omega$ : it is computed so that the maximal temperature at the final time is approximately of the order of $2000^{\circ} \mathrm{C}$, a typical value obtained with the more complete model of Section 3 .

\subsubsection{Shape optimization of the Von Mises stress}

In this context, the objective function $J_{1}(\Omega)$ for minimizing the Von Mises stresses reduces to

$$
J_{1}(\Omega)=\int_{0}^{t_{f}} \int_{\mathcal{D}}\left|\sigma_{D}\right|^{2} d x d t .
$$


We solve the optimization problem (48) with $\kappa=0.055$. The time discrete version of $J_{1}(\Omega)$ is

$$
J_{1}^{\Delta t}(\Omega)=\Delta t \sum_{j=1}^{N} \int_{\mathcal{D}}\left|2 \mu e\left(u_{j}\right)_{D}\right|^{2} d x,
$$

where $u_{j}$ satisfies (18). Note that in this case the objective function depends on the gradient of $u_{j}$, contrary to (19), so that the adjoint equation (20) has to be replaced by

$$
\begin{cases}-\operatorname{div}\left(A e\left(\eta_{j}\right)\right)=-\operatorname{div}\left(4 \mu \sigma_{D}^{j}\right) & \text { in } \mathcal{D} \\ \left(A e\left(\eta_{j}\right)\right) \cdot n=\left(4 \mu \sigma_{D}^{j}\right) \cdot n & \text { on } \partial \mathcal{D}_{F} \cup \partial \mathcal{D}_{N} \\ \eta_{j}=0 & \text { on } \partial \mathcal{D}_{D}\end{cases}
$$

The final shape after optimization is plotted in Figure 7 (bottom) where it can be compared with the initial shape (top). We observe in Figure 8 the decrease (by approximately 42,5\%) of the objective function, accounting for thermal stresses. In the mean time, compliance has increased only by the permitted $5.5 \%$ in the final optimized shape (see Figure 9). In Figure 10 one can check that the weight stays approximately constant. In Figures 11 are plotted the Von Mises norm of the stress for the initial (top) and final (bottom) shape, respectively. More precisely, we plot the following time average of the Von Mises stress for any point $x \in \mathcal{D}$

$$
\sqrt{\sum_{j=1}^{N} \Delta t\left|2 \mu e\left(u_{j}\right)_{D}\right|^{2}(x)}
$$

We observe that the shape optimization process reduces the zones of high thermal stresses from the initial shape $\Omega_{r e f}$ to the optimal shape $\Omega$.

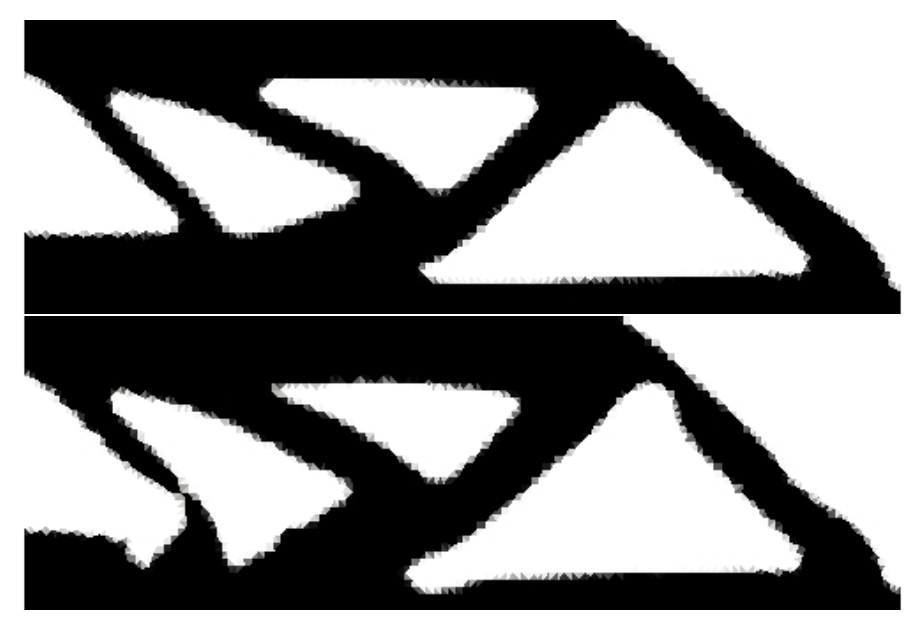

Figure 7: MBB beam initial (top) and final (bottom) shape for the test case of Section 5.2.1.

\subsubsection{Convergence study for time step refinement.}

The convergence of our optimization algorithm for time step refinement is studied. For the same optimization problem as in Section 5.2.1, we now divide the time step by two and multiply 


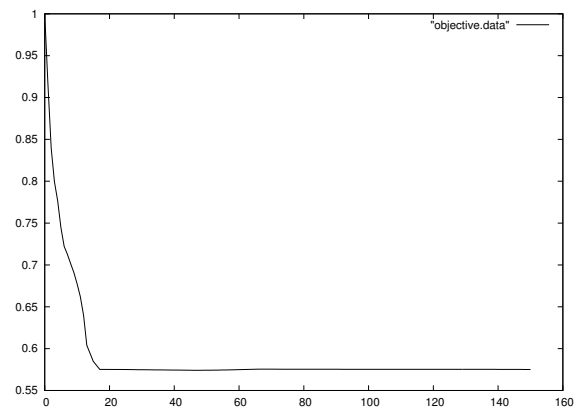

Figure 8: Convergence history for the objective function $J_{1}(\Omega)$ in the test case of Section 5.2.1.

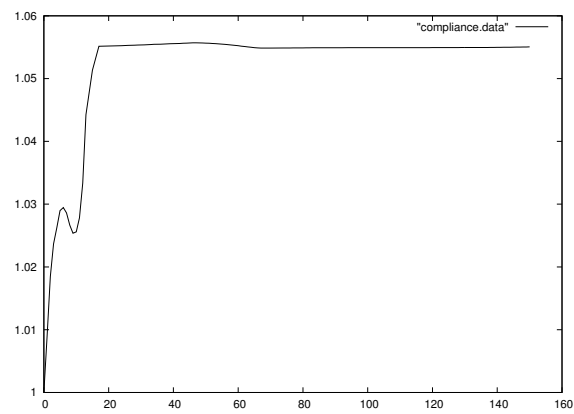

Figure 9: Convergence history for the compliance $C(\Omega)$ in the test case of Section 5.2.1.

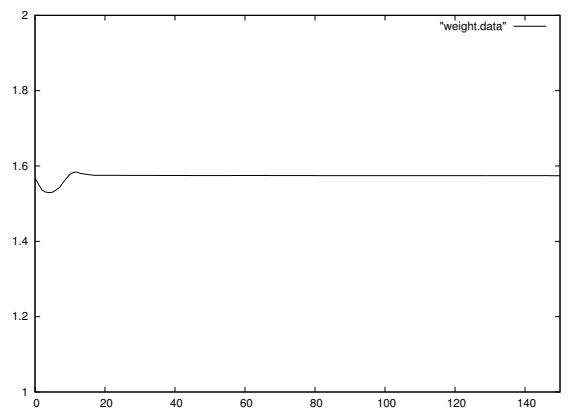

Figure 10: Convergence history for the weight $|\Omega|$ in the test case of Section 5.2.1.
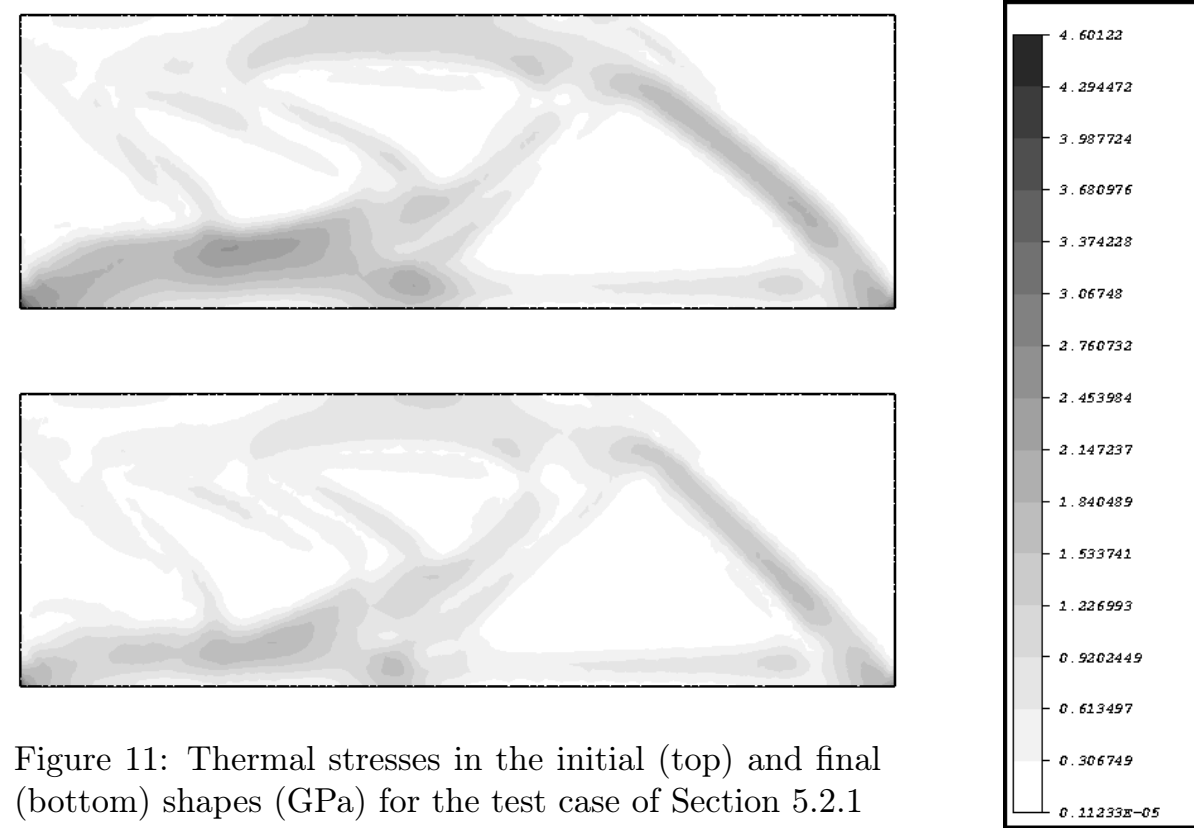

Figure 11: Thermal stresses in the initial (top) and final (bottom) shapes (GPa) for the test case of Section 5.2.1 
the number of time steps by two, so that the final time is still the same. In other words, we choose $\Delta t=0.05$ and $N=20$. In view of the results of Figure 12 our computation seems to converge under time step refinement, all other parameters being the same, including mesh size. The resulting optimized shapes are very similar. The only slight difference comes from the compliance constraint, which is slightly violated. This issue could be corrected by changing the values of the coefficients in the augmented Lagrangian algorithm, which we did not perform here for the sake of an unbiased comparison.
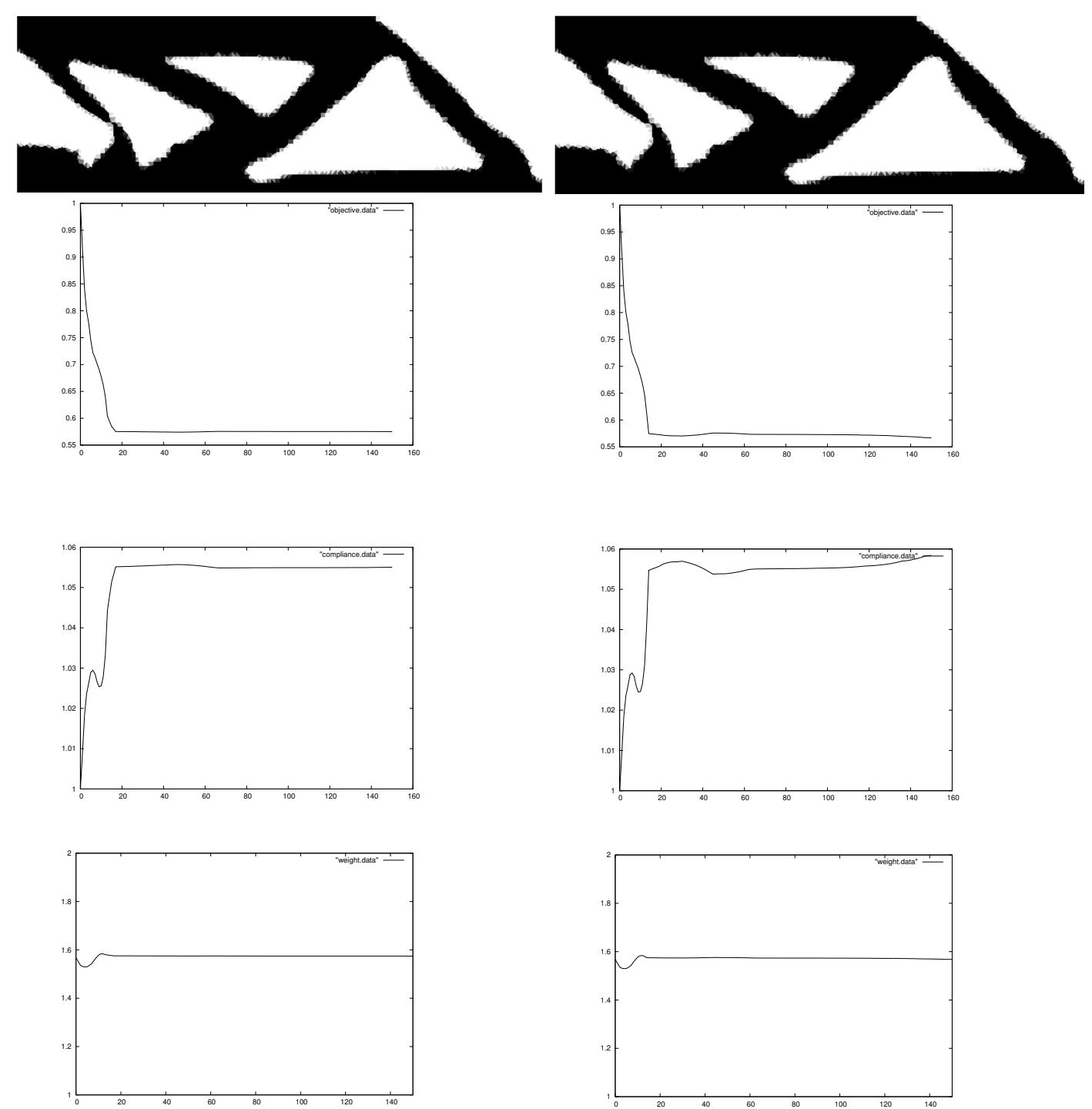

Figure 12: Test case of Section 5.2.2. From top to bottom: shape, objective function, compliance, weight. Left column: $\Delta t=0.1, N=10$. Right column: $\Delta t=0.05, N=20$. 


\subsubsection{Shape optimization of the vertical displacement}

For the simplified model of Section 2, the objective function $J_{2}(\Omega)$ is rewritten

$$
J_{2}(\Omega)=\int_{0}^{t_{f}} \int_{\mathcal{D}}\left|\max \left(0, u \cdot e_{d}-u^{+}\right)\right|^{2} d x d t .
$$

It amounts to minimize the vertical displacement, above a threshold $u^{+}$, everywhere and not merely in the top layer. We solve the optimization problem (48) with $\kappa=0.05$ and $u^{+}=0.005$. The time discrete version of $J_{2}(\Omega)$ is

$$
J_{2}^{\Delta t}(\Omega)=\Delta t \sum_{j=1}^{N} \int_{\mathcal{D}}\left|\max \left(0, u_{j} \cdot e_{d}-u^{+}\right)\right|^{2} d x
$$

where $u_{j}$ satisfies (18).

The optimized shape is displayed on Figure 13 (bottom) where it can be compared with the initial shape (top). We observe on Figure 14 that the objective function has decreased by approximately $47 \%$, while on Figure 15 we check that the compliance has increased by $5 \%$ at most, as required by the constraint in (48). As is confirmed by Figure 16 the weight stays approximately constant during the whole optimization process. In Figure 17 we make a comparison of the vertical displacement between the initial reference shape and the final optimized shape. More precisely, we plot a time average of the excess vertical displacement above the threshold $u^{+}$, for any point $x \in \mathcal{D}$,

$$
\sqrt{\sum_{j=1}^{N} \Delta t\left|\max \left(0, u_{j} \cdot e_{d}-u^{+}\right)\right|^{2}(x)} .
$$
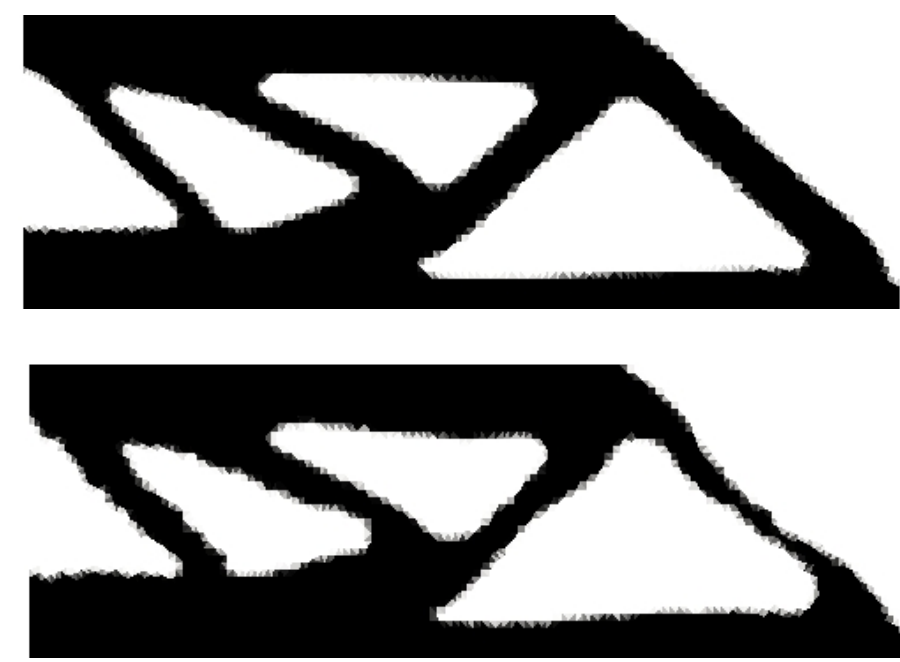

Figure 13: MBB beam initial (top) and final (bottom) shapes for the test case of Section 5.2.3.

\subsection{Layer by layer model of Section 3 in $2-d$}

We now consider the full layer by layer model of Section 3. It requires to solve the partial differential equations of our thermo-mechanical model in each intermediate domain $\mathcal{D}\left(t_{i}\right)$. This 


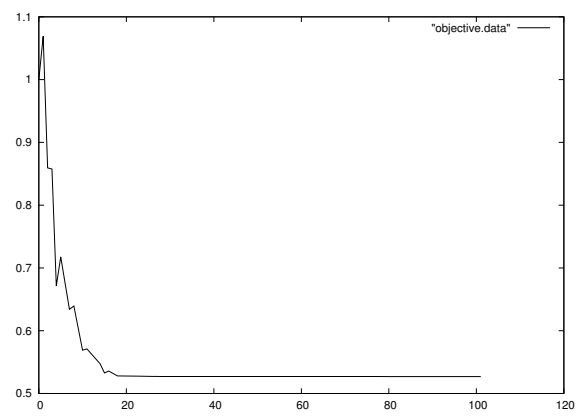

Figure 14: Convergence history for the objective function $J_{2}(\Omega)$ for the test case of Section 5.2.3

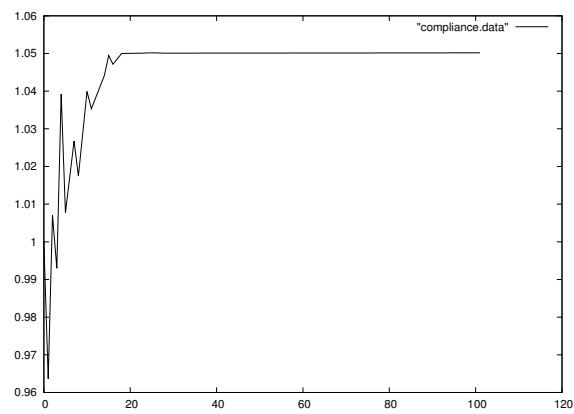

Figure 15: Convergence history for the compliance $C(\Omega)$ for the test case of Section 5.2.3

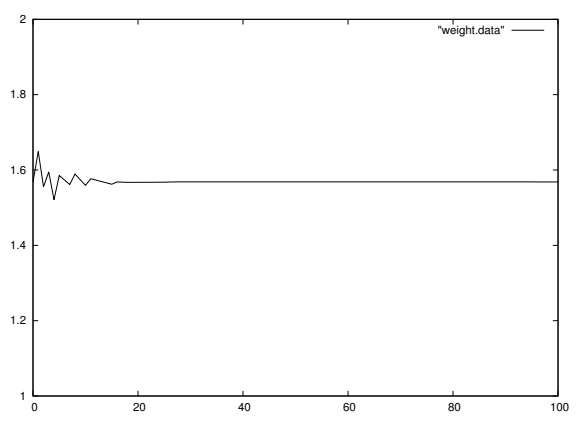

Figure 16: Convergence history for the weight $|\Omega|$ for the test case of Section 5.2.3
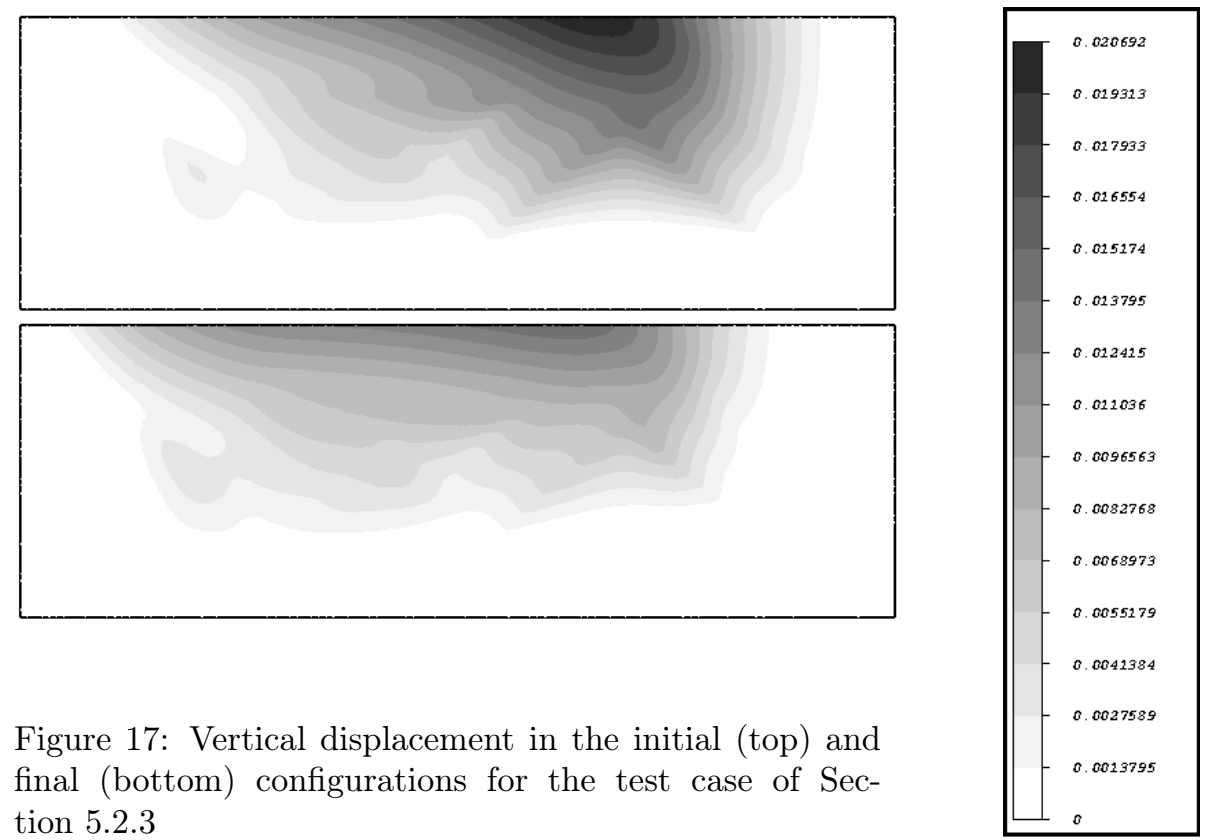

Figure 17: Vertical displacement in the initial (top) and final (bottom) configurations for the test case of Section 5.2 .3 
is possible if a new mesh is generated after adding a new layer. To avoid this book-keeping of several intermediate meshes, we rely on an ersatz material approach in a single mesh for the whole build chamber $\mathcal{D}$. More precisely, for each intermediate domain $\mathcal{D}\left(t_{i}\right)$, our model is solved in the full computational domain $\mathcal{D}$ where the "empty" space above $\mathcal{D}\left(t_{i}\right)$ is filled with a weak ersatz material. This ersatz material is defined by all its mechanical parameters being $10^{-6}$ smaller than the corresponding parameters of the solid and powder phases, except for its heat capacity which is, on the contrary, very large, equal to $\rho=10^{30}$. This last formula implies that, when solving the heat equation in $\mathcal{D}$, the temperature of the ersatz material will always be approximately equal to the initial temperature $T_{\text {init }}$. Whereas, the small mechanical parameters imply that the ersatz material play no role in the mechanical analysis of each $\mathcal{D}\left(t_{i}\right)$.

The laser is modeled by a heat source $Q$ moving in time in the last layer of each $\mathcal{D}\left(t_{i}\right)$. We consider the following parameters: $M=20$ layers, final time $t_{M}=1, \Delta t=0.01 \mathrm{~s}, N=5$, (so that in each layer there is 5 time steps) $Q_{1}=768000 \mathrm{~W} . \mathrm{cm}^{-2}, Q_{2}=10^{-3} Q_{1}$. Note that $Q_{1}$, applied in a cell of length $100 \mu \mathrm{m}$ and thickness $40 \mu \mathrm{m}$, corresponds to a laser power of $300 \mathrm{~W}$ with a thermal efficiency of precisely $10.24 \%$. For each layer of index $1 \leq i \leq M$, we define a sequence of cells $C_{i}^{j}$ of width $1 / N$ in the last layer $\mathcal{L}_{i}$ which are successively illuminated by the laser beam at each time step $t_{i}^{j}$, where $1 \leq j \leq N$ is the time index (see Figure 4). At time $t_{i}^{j}$, we apply a source term which is zero everywhere except in the cell $C_{i}^{j}$, where it takes the form

$$
Q\left(t_{i}^{j}, x\right)=Q_{1} \chi_{\Omega}(x)+Q_{2} \chi_{\mathcal{P}}(x)
$$

The other parameters are $\epsilon=\varepsilon=2 \Delta x$, with a mesh size approximately $\Delta x=0.04$. The beam trajectory is a sequence of horizontal paths from left to right, as can be seen on Figure 18 where the temperature field is plotted during the building process for the reference shape of Figure 6 : from top to bottom, snapshots at times $t_{3}+\Delta t, t_{7}+2 \Delta t, t_{13}+3 \Delta t, t_{15}+\Delta t, t_{19}+2 \Delta t$.

\subsubsection{Shape optimization of the Von Mises stress in the layer by layer construction}

In this case the objective function is $J_{1}(\Omega)$, defined by $(46)$, and we solve the optimization problem (48) with $\kappa=0.09$. The time discrete version of $J_{1}(\Omega)$ is

$$
J_{1}^{\Delta t}(\Omega)=\sum_{i=1}^{M} \Delta t \sum_{j=1}^{N} \int_{\mathcal{D}\left(t_{i}\right)}\left|\left(\sigma_{i}^{j}\right)_{D}\right|^{2} d x
$$

where $\sigma_{i}^{j}$ is the solution of (37).

In Figure 19, we compare the final shape after optimization (bottom) with the initial shape (top). In Figure 20, the following time average of the thermal stress is plotted

$$
\sqrt{\sum_{i=1}^{M} \Delta t \sum_{j=1}^{N}\left|2 \mu e\left(u_{i}^{j}\right)_{D}\right|^{2}(x)}, \quad x \in \mathcal{D},
$$

for both initial (top) and final (bottom) shapes. One can clearly see a reduction of the zones of high thermal stress from the initial shape $\Omega_{\text {ref }}$ to the optimal shape $\Omega$. As can be checked on Figure 21, the objective function has decreased by approximately $27 \%$, while on Figure 22 the compliance has increased by $9 \%$ as permitted by the constraint in (48). Figure 23 shows that the weight stays approximately constant. 

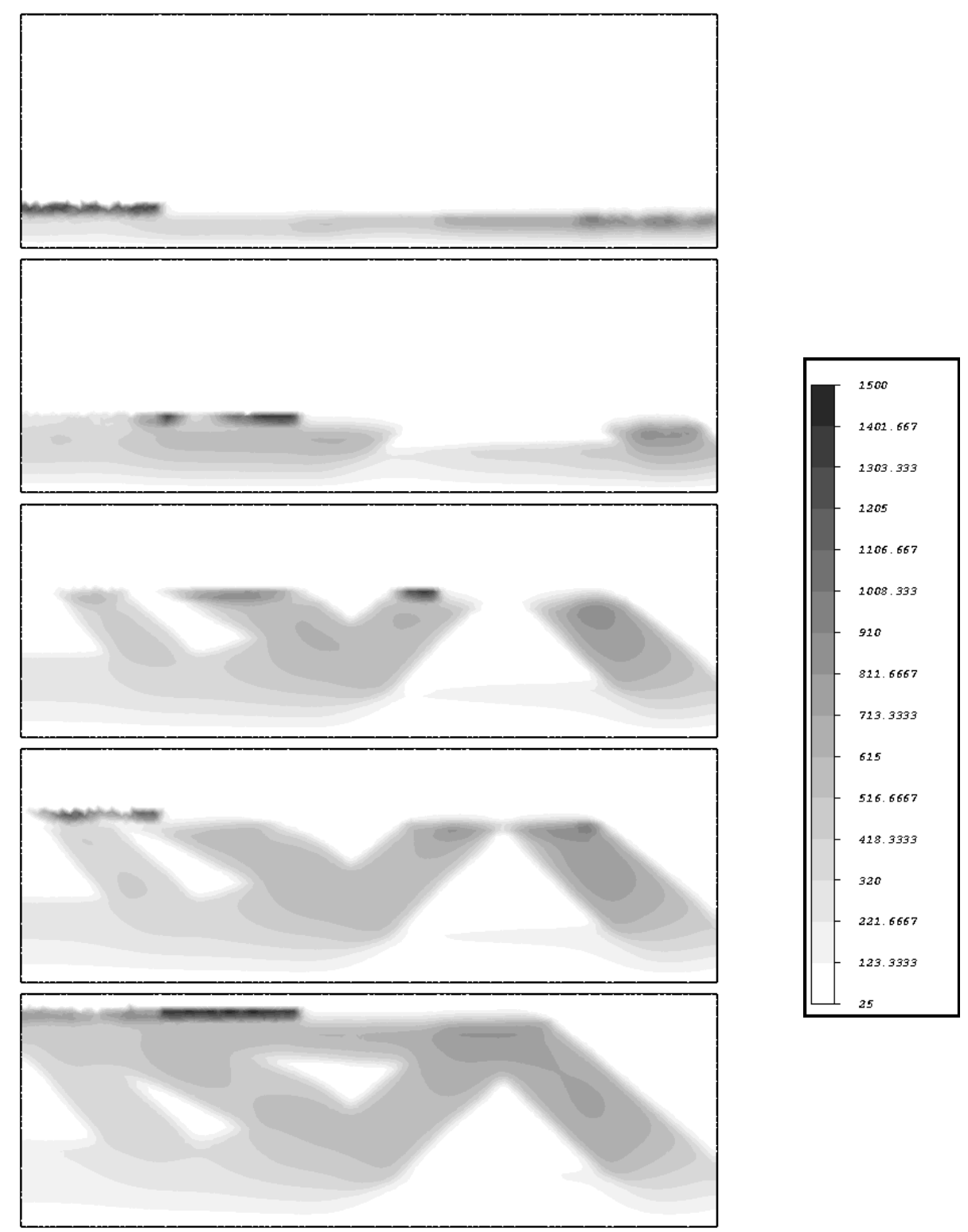

Figure 18: Temperature evolution during the building process for the layer by layer model of Section 3 at times $t_{3}+\Delta t, t_{7}+2 \Delta t, t_{13}+3 \Delta t, t_{15}+\Delta t, t_{19}+2 \Delta t$. 

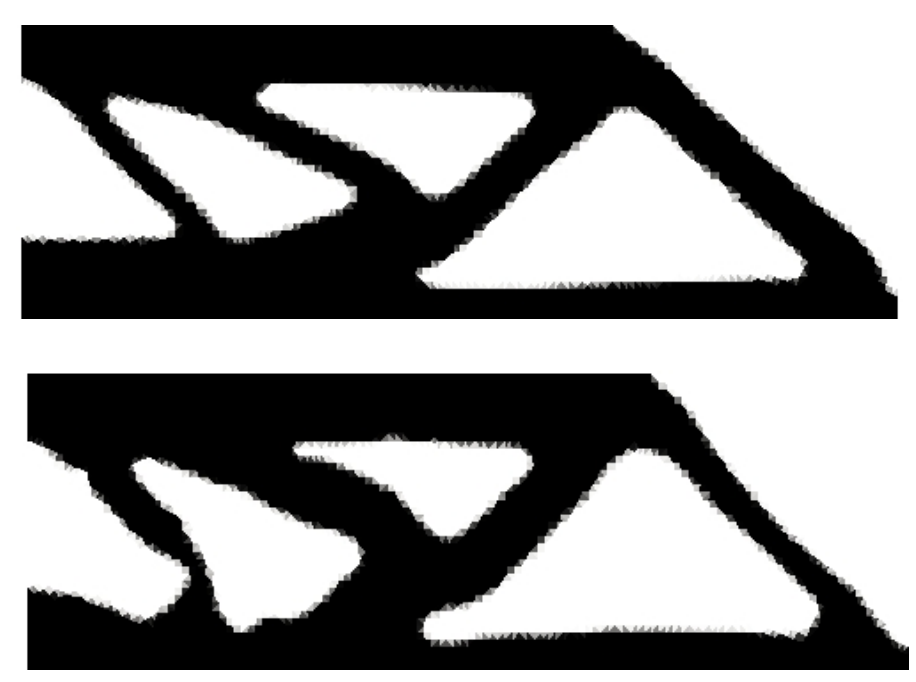

Figure 19: BBM beam initial (top) and final (bottom) shapes for the test case of Section 5.3.1.

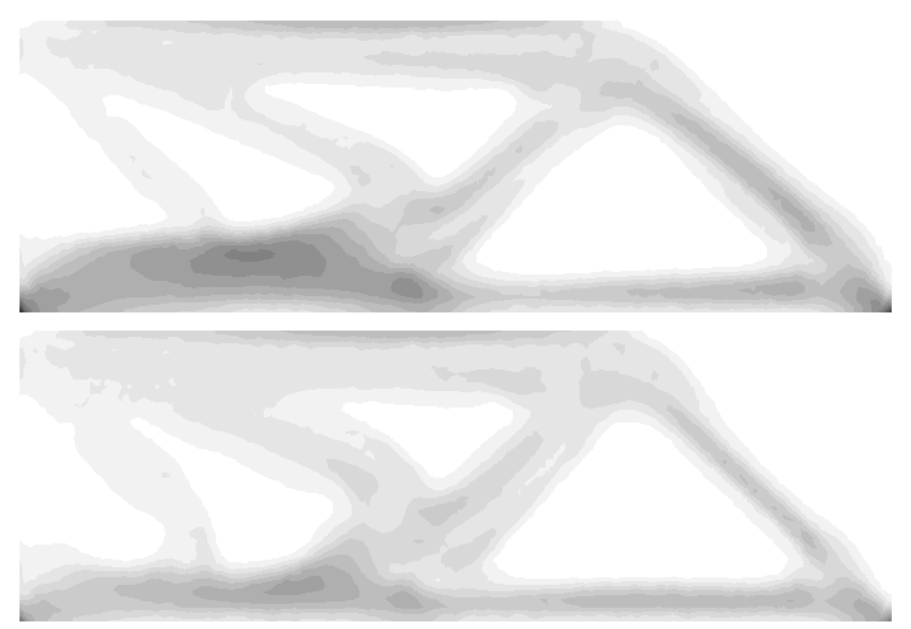

Figure 20: Thermal stresses in the initial (top) and final (bottom) configurations (GPa) for the test case of Section 5.3.1.

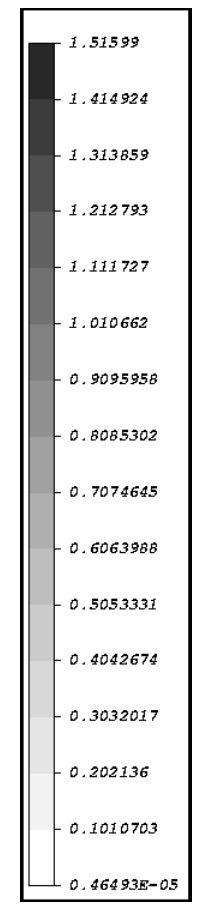




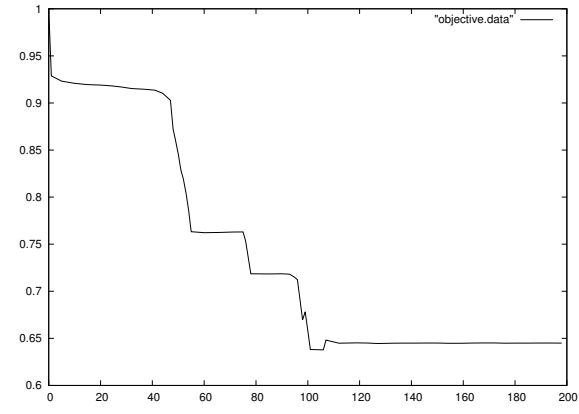

Figure 21: Convergence history for the objective function $J_{1}(\Omega)$ for the test case of Section 5.3.1.

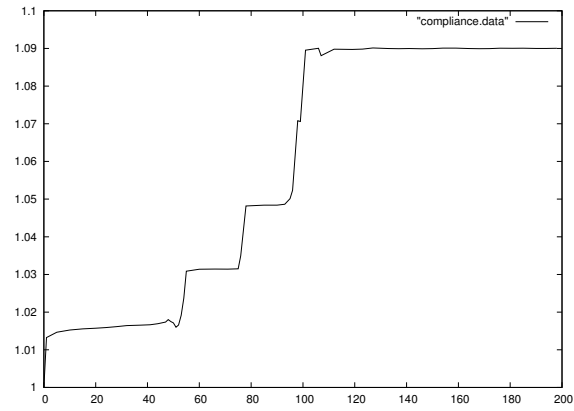

Figure 22: Convergence history for the compliance $C(\Omega)$ for the test case of Section 5.3.1.

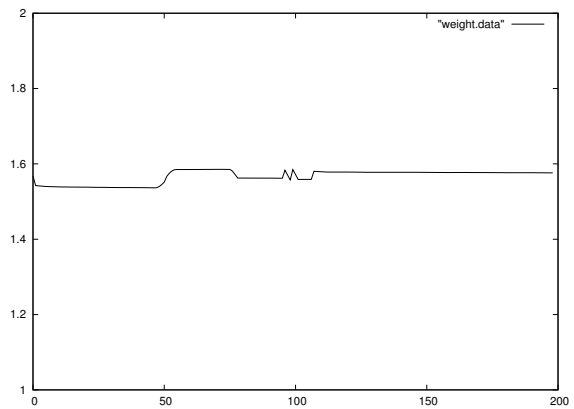

Figure 23: Convergence history for the weight $|\Omega|$ for the test case of Section 5.3.1. 


\subsubsection{Shape optimization of the vertical displacement in the layer by layer con- struction}

In this case the objective function is $J_{2}(\Omega)$, defined by $(47)$, and we solve the optimization problem (48) with $\kappa=0.05$. The time discrete version of $J_{2}(\Omega)$ is

$$
J_{2}^{\Delta t}(\Omega)=\sum_{i=1}^{M} \Delta t \sum_{j=1}^{N} \int_{\mathcal{D}\left(t_{i}\right) \backslash \mathcal{D}\left(t_{i-1}\right)}\left|\max \left(0, u_{i}^{j} \cdot e_{d}-u^{+}\right)\right|^{2} d x
$$

where $u_{i}^{j}$ is the solution of (37) and $u^{+}=0.005$.

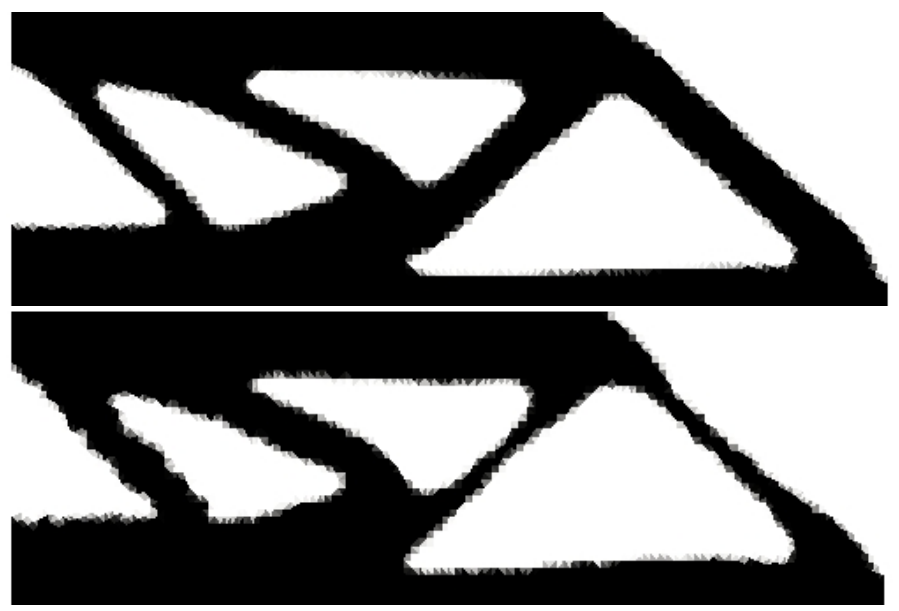

Figure 24: BBM beam initial (top) and final (bottom) shapes for the test case of Section 5.3.2.

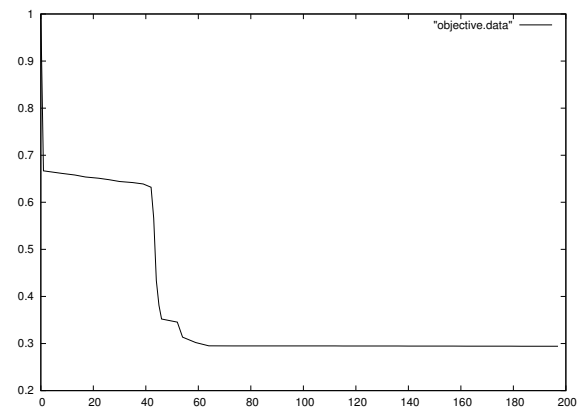

Figure 25: Convergence history for the objective function $J_{2}(\Omega)$ for the test case of Section 5.3.2.

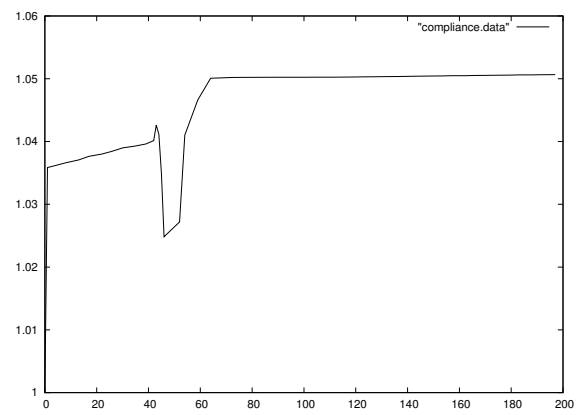

Figure 26: Convergence history for the compliance $C(\Omega)$ for the test case of Section 5.3.2.

In Figure 24, we compare the initial (top) and final (bottom) shapes after optimization. Figure 25 shows that the objective function $J_{2}$ (measuring the excess vertical displacement in the last layer of each intermediate structure) has decreased by approximately $70 \%$. On the other hand, the compliance has increased by no more than $5 \%$, as required by the constraint in 


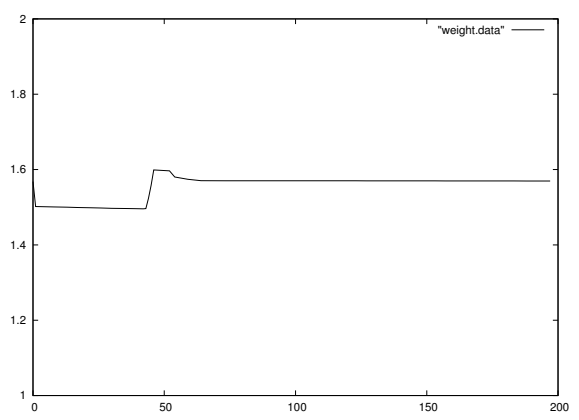

Figure 27: Convergence history for the weight $|\Omega|$ for the test case of Section 5.3.2.
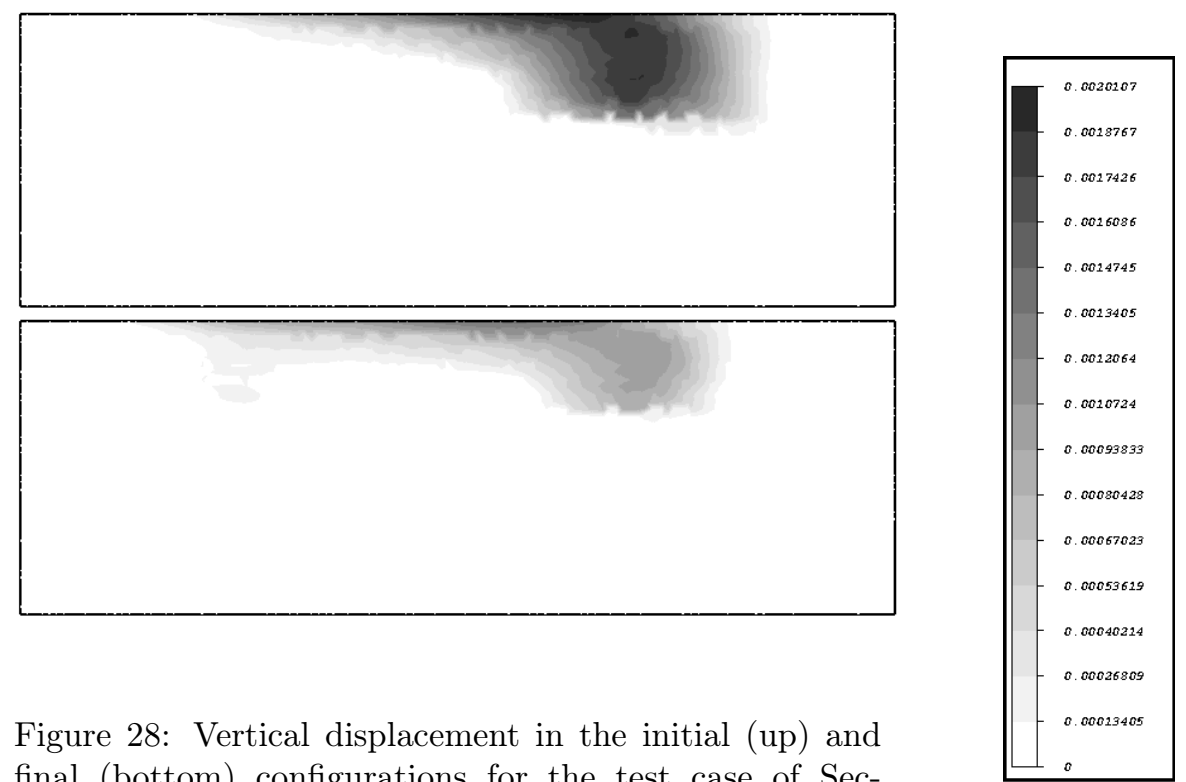

Figure 28: Vertical displacement in the initial (up) and final (bottom) configurations for the test case of Section 5.3.2. 
(48) (see Figure 26). One can check on Figure 27 that the weight stays approximately constant. In Figure 28 we plot the norm of the excess vertical displacement in each top layers of the intermediate shapes, namely the following quantity

$$
\sqrt{\sum_{i=1}^{M} \Delta t \chi_{\mathcal{L}_{i}}(x) \sum_{j=1}^{N}\left|\max \left(0, u_{i}^{j} \cdot e_{d}-u^{+}\right)\right|^{2}(x)}, \quad x \in \mathcal{D} .
$$

Clearly, one can see a reduction of the zones of excess vertical displacement between the initial (top) and final (bottom) shapes in Figure 28.

\subsection{A 3-d case for the simplified model of Section 2}

For minimizing the computational time in 3-d, we consider only the simplified model of Section 2 with the following parameters: final time $t_{f}=1, \Delta t=0.1 \mathrm{~s}, N=10, Q_{1}=8000 \mathrm{~W} . \mathrm{cm}^{-3}$, $Q_{2}=10^{-3} Q_{1}$, and $\epsilon=\varepsilon=2 \Delta x$ with a mesh size of approximately $\Delta x=0.09$. The value of the source term $Q_{1}$ does not correspond to the laser power since it is applied in the full final shape $\Omega$ : it is computed so that the maximal temperature at the final time is approximately of the order of $2000^{\circ} \mathrm{C}$, a typical value obtained with the more complete model of Section 3 .

\subsubsection{Shape optimization of the Von Mises stress}

The objective function $J_{1}(\Omega)$ for minimizing the Von Mises stresses is, as in Section 5.2.1,

$$
J_{1}(\Omega)=\int_{0}^{t_{f}} \int_{\mathcal{D}}\left|\sigma_{D}\right|^{2} d x d t
$$

We solve the optimization problem (48) with $\kappa=0.08$. As usual, the initial and reference shape $\Omega_{\text {ref }}$ is obtained by a simple minimal compliance problem without any manufacturing constraint. On Figure 29 one can compare the initial shape (left) and the final one (right). Each column in Figure 29 displays the same shape, viewed from two different orientations.

We observe in Figure 30 that the objective function has decreased by approximately $48 \%$, while the compliance has not increased more than the allowed $8 \%$ for the final optimized shape (Figure 31). In Figure 32 one can check that the weight converges, as required, to the initial weight at the end of the optimization process. In Figure 33, we plot the thermal stresses, as defined by (49), in a vertical cross section $(y=0.7 \mathrm{~cm})$ of the initial and final shapes. One can observe, as in $2-\mathrm{d}$, that the optimization process reduces the zones where the thermal stresses are large.

\subsubsection{Shape optimization of the vertical displacement}

The objective function $J_{2}(\Omega)$, for minimizing the vertical displacement, reduces as in 2-d to

$$
J_{2}(\Omega)=\int_{0}^{t_{f}} \int_{\mathcal{D}}\left|\max \left(0, u \cdot e_{d}-u^{+}\right)\right|^{2} d x d t .
$$

We solve the optimization problem (48) with $\kappa=0.08$ and $u^{+}=0.005$.

In Figure 34 the final shape after optimization (right) is plotted and compared to the initialization (left). Each column in Figure 34 displays the same shape, viewed from two different orientations. We observe in Figure 35 that the objective function (measuring the vertical displacements) has decreased by approximately $70 \%$, while the compliance has increased only by 

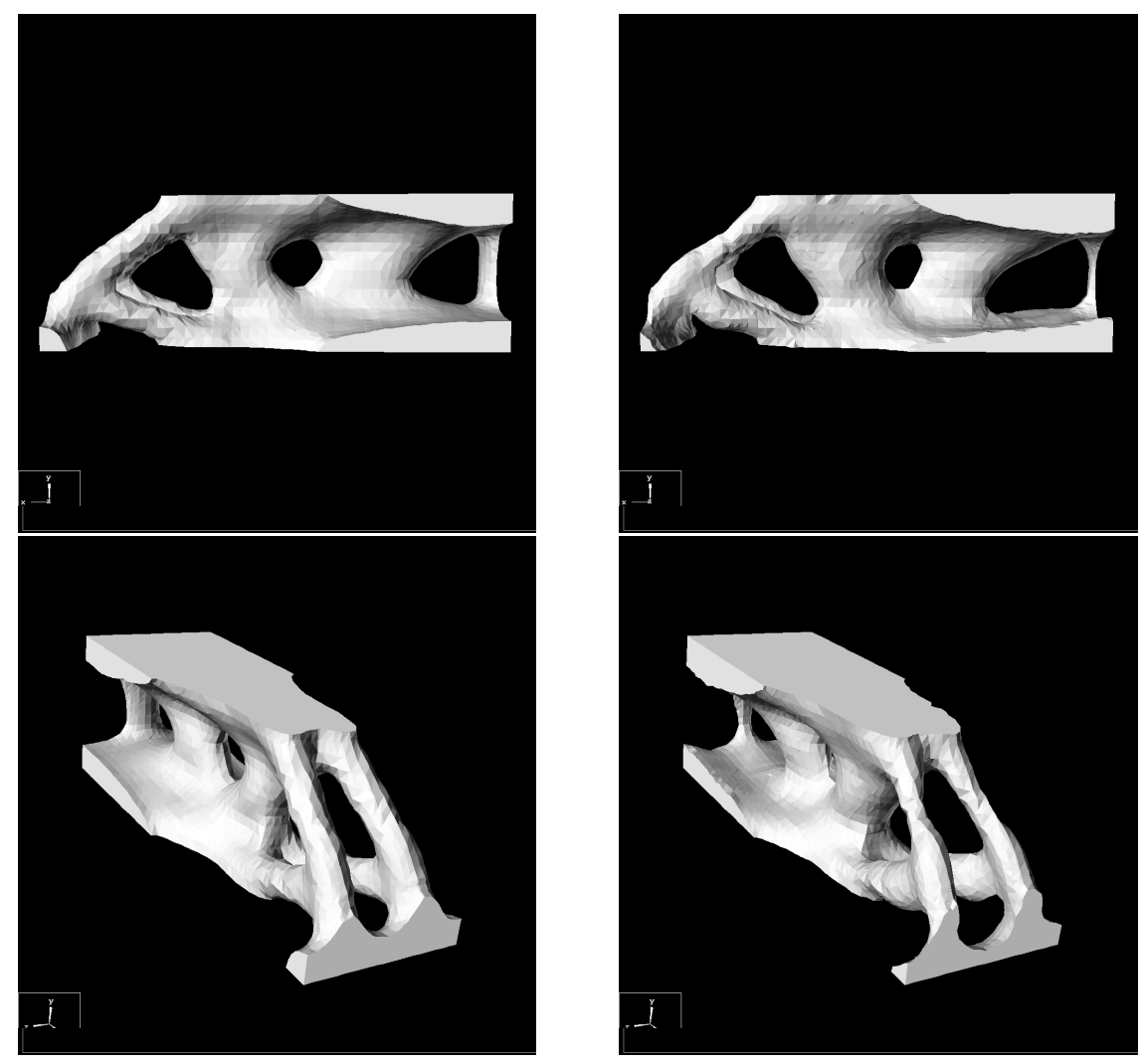

Figure 29: Initial (left) and final (right) configuration for the test case of Section 5.4.1.

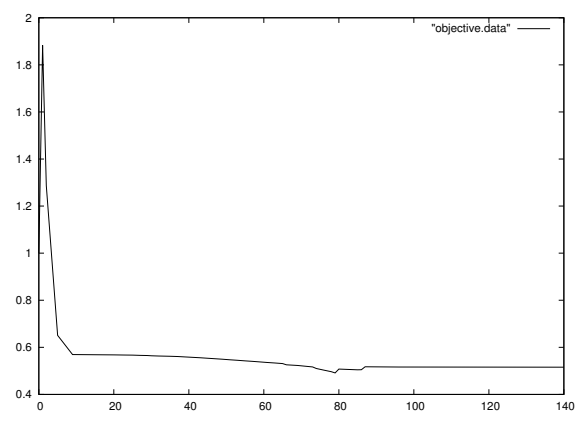

Figure 30: Convergence history for the objective function $J_{1}(\Omega)$ for the test case of Section 5.4.1.

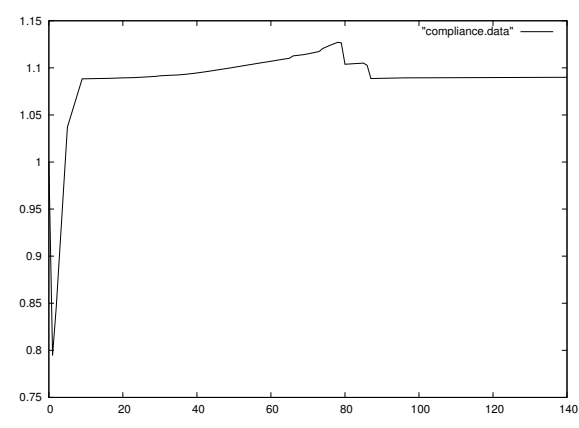

Figure 31: Convergence history for the compliance $C(\Omega)$ for the test case of Section 5.4.1. 


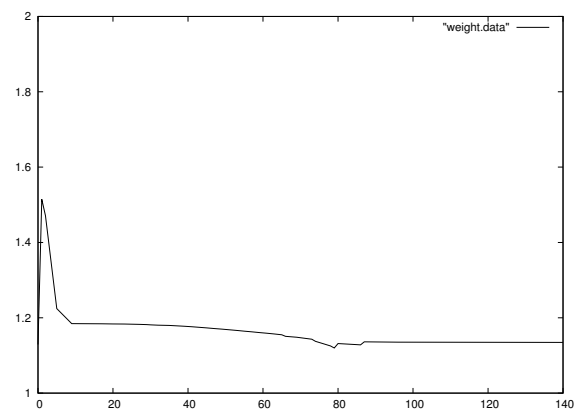

Figure 32: Convergence history for the weight $|\Omega|$ for the test case of Section 5.4.1.

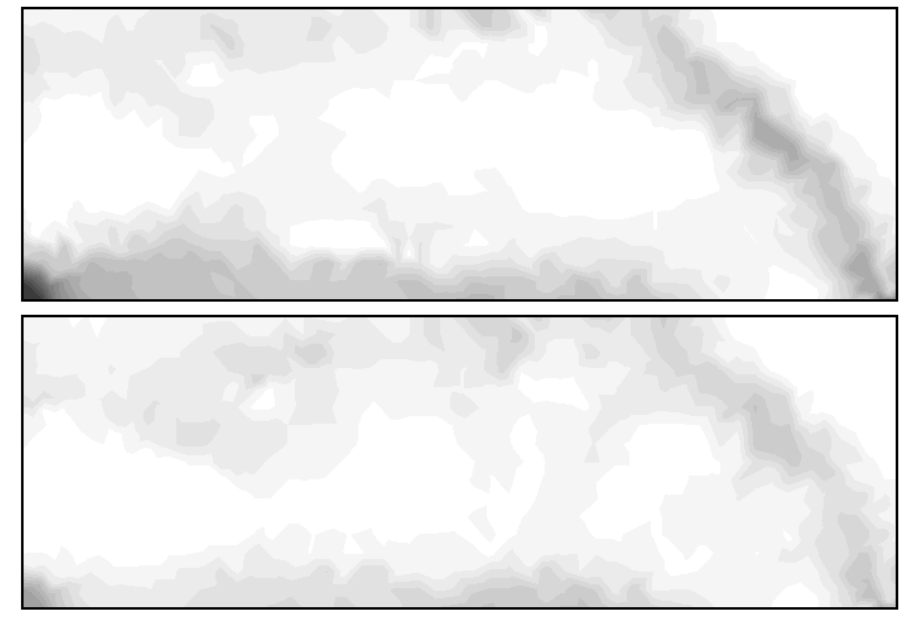

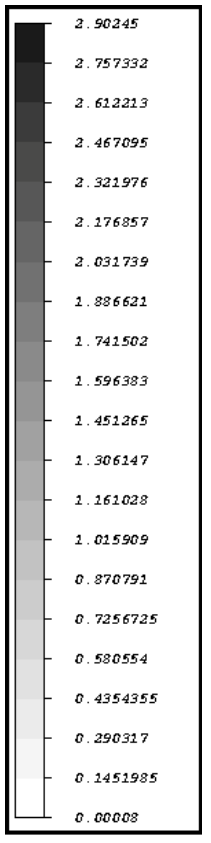

Figure 33: Thermal stresses (GPa) in the initial (top) and final (bottom) configurations for the test case of Section 5.4.1, vertical cross section $y=0.7 \mathrm{~cm}$.

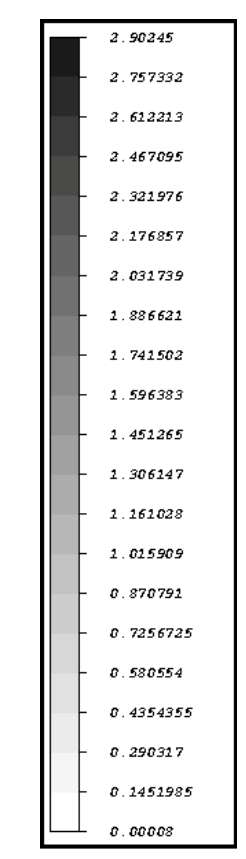



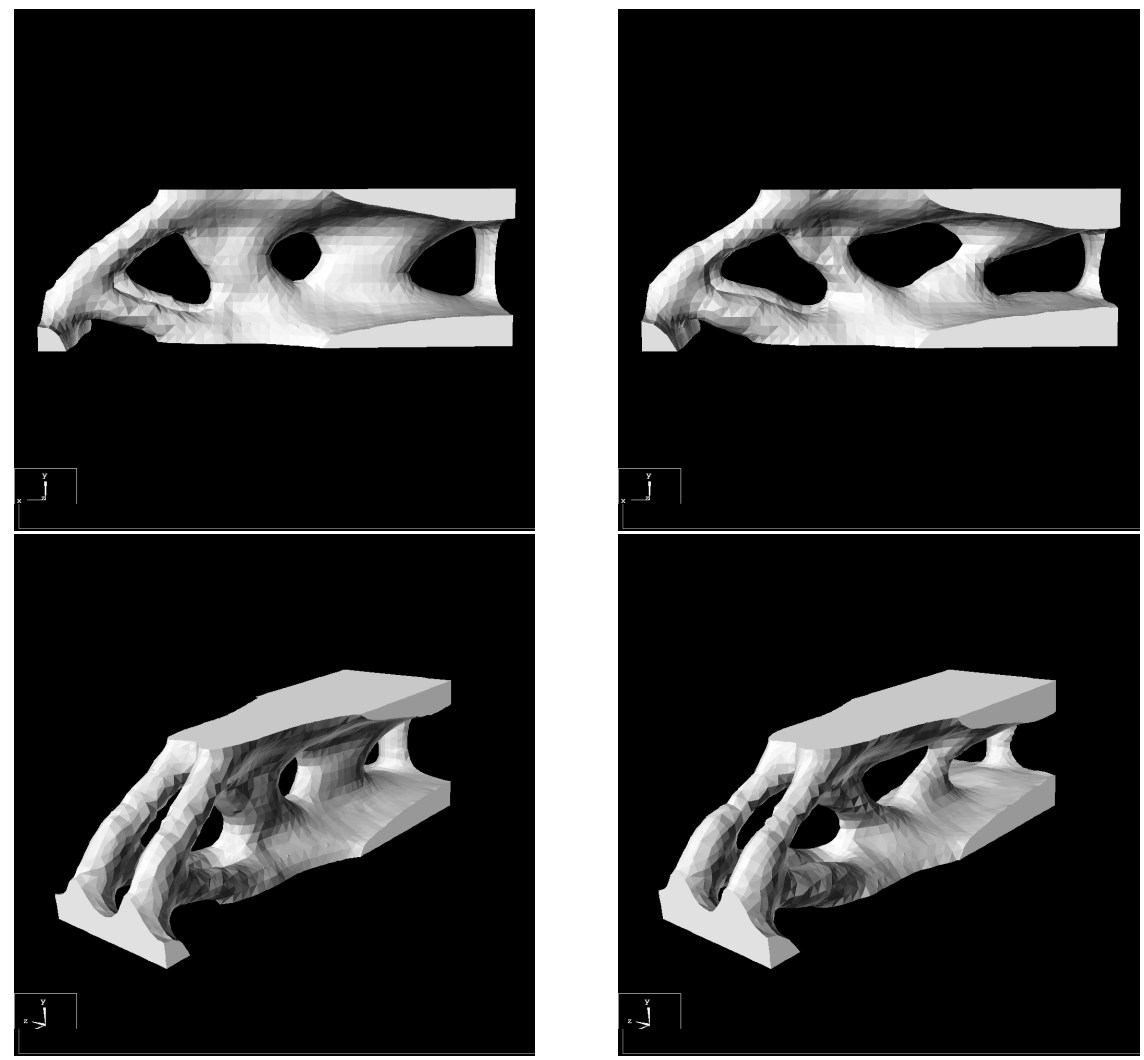

Figure 34: Initial (left) and final (right) shapes for the optimization of Section 5.4.2.

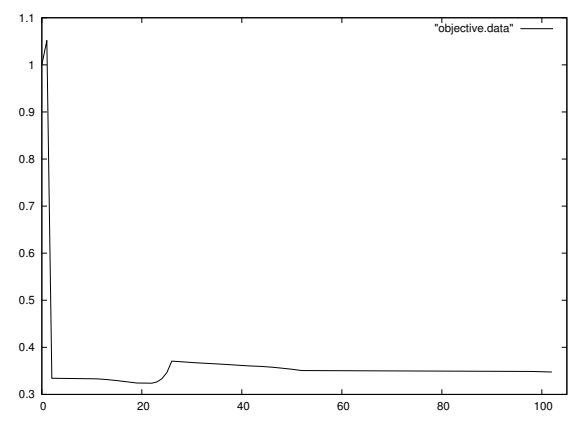

Figure 35: Convergence history for the objective function $J_{2}(\Omega)$ in the test case of Section 5.4.2.

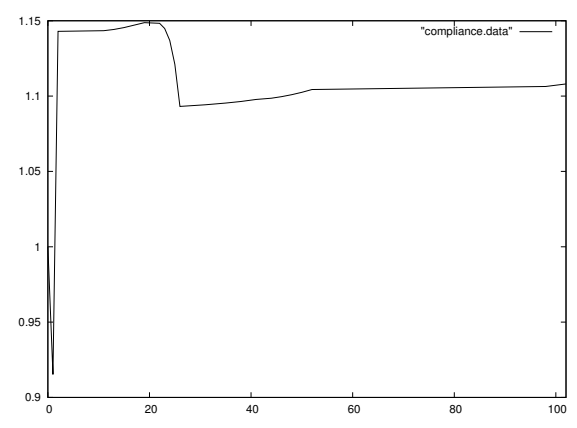

Figure 36: Convergence history for the compliance $C(\Omega)$ in the test case of Section 5.4.2. 


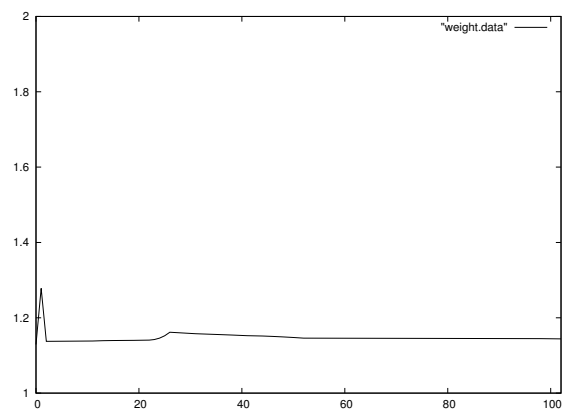

Figure 37: Convergence history for the weight $|\Omega|$ in the test case of Section 5.4.2.

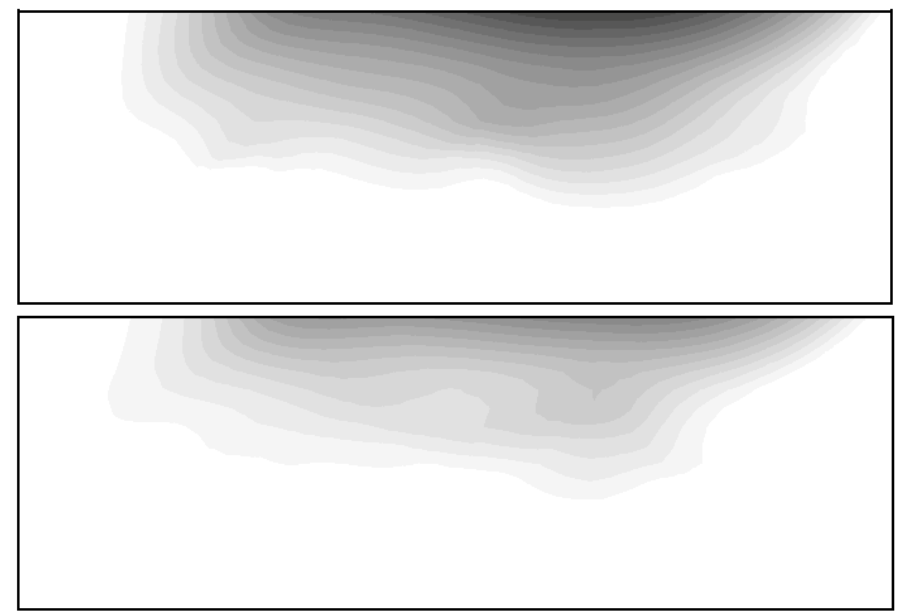

\begin{tabular}{|l|l|}
\hline 0.020924 \\
0.0098882 \\
0.009292 \\
0.0087757 \\
0.0082595 \\
0.0077493 \\
-0.0072271 \\
0.0067109 \\
0.0061946 \\
0.0056784 \\
\hline 0.0051622 \\
\hline 0.004646 \\
\hline 0.0042998 \\
0.0036135 \\
\hline 0.0030973 \\
\hline 0.0025812 \\
\hline 0.0020649 \\
\hline 0.0015487 \\
\hline 0.0010324 \\
\hline 0.00051622 \\
\hline 0 \\
\hline
\end{tabular}

Figure 38: Vertical displacements in the initial (top) and final (bottom) shapes for the optimization in Section 5.4.2, vertical cross section $y=0.7 \mathrm{~cm}$. 
approximately $8-10 \%$ in the final optimized shape (see Figure 36 ). One can check on Figure 37 that the weight converges to the required initial weight during the optimization process. The excess vertical displacement, as evaluated by (50), is plotted on Figure 38, in a vertical cross section $(y=0.7 \mathrm{~cm})$ for the initial (top) and final (bottom) shapes. Once again, the zones of high excess vertical displacement have decreased in size during the optimization process.

\section{Conclusion}

In this paper we proposed a thermo-mechanical model, coupled to the heat equation, for evaluating the behavior of a structure during its building process by additive manufacturing techniques. Two variants have been tested: a complete layer by layer model which is precise but computationally expensive, a simplified model working directly on the final shape which is computationally cheap but does not take into account the building history. Two objective functions have been tested: first, a measure of the thermal residual stress and, second, a least square penalization of the excess vertical displacement in the top layers. All models and objective functions perform very well in our shape and topology optimization, in 2-d and 3-d.

What remains to be done is the following. First, for more industrial examples, finer meshes and time discretizations should be tried. Of course, it will require more computational power. Other objective functions could also be tested, which does not require any further theoretical developments. Eventually, our modeling of thermal residual stress is quite crude in the absence of any plasticity effects. In a future work, we plan to add plastic irreversible deformations in our thermo-mechanical model, following the original model of [42].

\section{Acknowledgements}

L. J. is a post-doctoral researcher, funded by the DGA (Direction Générale de l'Armement), the support of which is kindly acknowledged. G. A. is a member of the DEFI project at INRIA Saclay Ile-de-France. The work of G. A. is partially supported by the SOFIA project, funded by BPI (Banque Publique d'Investissement). Both authors thank François Jouve for his help with the graphic tool $\mathrm{xd} 3 \mathrm{~d}$.

\section{References}

[1] G. Allaire, Ch. Dapogny, G. Delgado, G. Michailidis, Multi-phase structural optimization via a level set method, COCV 20, pp.576-611 (2014).

[2] G. Allaire, Ch. Dapogny, A. Faure, G. Michailidis, Shape optimization of a layer by layer mechanical constraint for additive manufacturing, C. R. Math. Acad. Sci. Paris, 355, no. 6, 699-717 (2017).

[3] G. Allaire, C. Dapogny, R. Estevez, A. Faure and G. Michailidis, Structural optimization under overhang constraints imposed by additive manufacturing technologies, Journal of Computational Physics, 351, 295-328 (2017).

[4] G. Allaire, F. Jouve, G. Michailidis, Thickness control in structural optimization via a level set method, SMO 53, 1349-1382 (2016). 
[5] G. Allaire, F. Jouve, G. Michailidis, Molding direction constraints in structural optimization via a level-set method, in Variational Analysis and Aerospace Engineering, Mathematical Challenges for the Aerospace of the Future, Frediani, A., Mohammadi, B., Pironneau, O., Cipolla, V. (Eds.), Springer Optimization and Its Applications 116, pp.1-39, Springer (2016).

[6] G. Allaire, F. Jouve, A.-M. Toader, Structural optimization using sensitivity analysis and a level-set method, J. Comp. Phys. Vol 194/1, pp.363-393 (2004).

[7] G. Allaire, F. Jouve, N. Van Goethem, Damage and fracture evolution in brittle materials by shape optimization methods, J. Comp. Phys. 230, 5010-5044 (2011).

[8] C. Barlier, A. Bernard, Fabrication additive - Du Prototypage Rapide à l'impression 3D, Dunod, Paris (2016).

[9] A. Bastier, M.H. Maitournam, F. Roger, K. Dang Van Modelling of the residual state of friction stir welded plates. Journal of materials processing technology 200, 25-37, (2008).

[10] Ch. Bernardi, O. Pironneau, Sensitivity of Darcy's law to discontinuities, Chinese Ann. Math. Ser. B 24, no. 2, pp.205-214 (2003).

[11] S. Cacace, E. Cristiani, L. Rocchi, A level set based method for fixing overhangs in 3D printing, Applied Mathematical Modelling, 44, 446-455 (2017).

[12] J. Céa, Conception optimale ou identification de formes, calcul rapide de la dérivée directionnelle de la fonction coût, Math. Model. Num. Anal. 20, 3, 371-402 (1986).

[13] C. Dapogny, A. Faure, G. Michailidis, G. Allaire, A. Couvelas, R. Estevez, Geometric constraints for shape and topology optimization in architectural design, Computational Mechanics, 59, no. 6, 933-965 (2017).

[14] C. Dapogny, P. Frey, Computation of the signed distance function to a discrete contour on adapted triangulation, Calcolo, Volume 49, Issue 3, 193-219 (2012).

[15] O. Fergani, F. Berto, T. Welo, S.Y. Liang, Analytical modelling of residual stress in additive manufacturing, Fatigue Fract Engng Mater Struct, 40: 971-978 (2017).

[16] I. Gibson, D. W. Rosen, B. Stucker, Additive manufacturing technologies, Springer, New York (2010).

[17] J.K. Guest, J.H. Prévost, T. Belytschko, Achieving minimum length scale in topology optimization using nodal design variables and projection functions, International Journal for Numerical Methods in Engineering, 61(2), 238-254 (2004).

[18] J.K. Guest, Imposing maximum length scale in topology optimization, Structural and Multidisciplinary Optimization, 37(5), 463-473 (2009).

[19] X. Guo, W. Zhang, W. Zhong, Explicit feature control in structural topology optimization via level set method, Computer Methods in Applied Mechanics and Engineering, 272(0), 354-378 (2014).

[20] A.V. Gusarov, I. Yadroitsev, Ph. Bertrand, I. Smurov, Model of radiation and heat transfer in laser-powder interaction zone at selective laser melting, J. Heat Transf., 131(7):072101 (2009). 
[21] L. Harzheim, G. Graf, A review of optimization of cast parts using topology optimization: I - topology optimization with manufacturing constraints, Structural and multidisciplinary optimization, 31(5), 388-399 (2006).

[22] F. Hecht, New development in FreeFem ++, J. Numer. Math. 20, no. 3-4, 251-265 (2012).

[23] J.C. Heigel, P. Michaleris, E.W. Reutzel, Thermo-mechanical model development and validation of directed energy deposition additive manufacturing of Ti-6Al-4V, Additive Manufacturing 5, 9-19 (2015).

[24] F. Hettlich, W. Rundell, The determination of a discontinuity in a conductivity from a single boundary measurement, Inverse Problems, 14, no. 1, pp.67-82 (1998).

[25] N. E. Hodge, R. M. Ferencz, J. M. Solberg, Implementation of a thermomechanical model for the simulation of selective laser melting, Comput. Mech. 54, no. 1, 33-51 (2014).

[26] M. Langelaar, An additive manufacturing filter for topology optimization of print-ready designs, Structural and Multidisciplinary Optimization, 1-13 (2016).

[27] M. Langelaar, Topology optimization of 3D self-supporting structures for additive manufacturing, Additive Manufacturing, 12, 60-70 (2016).

[28] M. Leary, L. Merli, F. Torti, M. Mazur, M. Brandt, Optimal topology for additive manufacture: a method for enabling additive manufacture of support-free optimal structures, Materials \& Design, 63, 678-690 (2014).

[29] J.L. Lions, E. Magenes, Problèmes aux limites non homogènes (3 volumes), Dunod, Paris (1968).

[30] M. Megahed, H.-W. Mindt, N. N'Dri, H. Duan, O. Desmaison, Metal additive-manufacturing process and residual stress modeling, Integrating Materials and Manufacturing Innovation, $5: 4,(2016)$.

[31] F. Murat, J. Simon, Etudes de problèmes d'optimal design. Lecture Notes in Computer Science 41, 54-62, Springer Verlag, Berlin (1976).

[32] S. Osher, J.A. Sethian, Front propagating curvature dependent speed: algoritms based on Hamilton-Jacobi formulations, JCP, 78, 12-49, (1988).

[33] O. Pantz, Sensibilité de l'équation de la chaleur aux sauts de conductivité, C. R. Acad. Sci. Paris, Ser. I 341 pp.333-337 (2005).

[34] O. Pironneau, Optimal shape design for elliptic systems, Springer-Verlag, New York, (1984).

[35] I.A. Roberts, C.J.Wang, R. Esterlein, M. Stanford, D.J. Mynors, A three-dimensional finite element analysis of the temperature field during laser melting of metal powders in additive layer manufacturing, International Journal of Machine Tools and Manufacture, 49(12-13), 916-923 (2009).

[36] B. Schoinochoritis, D. Chantzis, K.Salonitis, Simulation of metallic powder bed additive manufacturing processes with the finite element method: A critical review. Journal of Engineering Manufacture, 1-22, (2015).

[37] O. Sigmund, Manufacturing tolerant topology optimization, Acta Mechanica Sinica, 25(2), 227-239 (2009). 
[38] O. Sigmund, S. Torquato, Design of materials with extreme thermal expansion using a threephase topology optimization method, J. Mech. Phys. Solids 45, no. 6, 1037-1067, (1997).

[39] J. Sokolowski, J.-P. Zolesio, Introduction to shape optimization: shape sensitivity analysis, Springer Series in Computational Mathematics, Vol.10, Springer, Berlin (1992).

[40] M. Suard, P. Lhuissier, R. Dendievel, J.-J. Blandin, F. Vignat, F. Villeneuve, Towards stiffness prediction of cellular structures made by electron beam melting (EBM), Powder Metallurgy, 57(3), 190-195 (2014).

[41] L. Van Belle, Analyse, modélisation et simulation de l'apparition de contraintes en fusion laser métallique. Thèse de Doctorat, INSA de Lyon (2013).

[42] L. Van Belle, J.-C. Boyer, G. Vansteenkiste, Investigation of residual stresses induced during the selective laser melting process. Key Engineering Materials, 1828-2834, (2013).

[43] A. S. Wu, D. W. Brown, M. Kumar, G. F. Gallegos, W. E. King, An experimental investigation into additive manufacturing-induced residual stresses in 316L stainless steel, Metallurgical and Materials Transactions A, 45(13), 6260-6270 (2014).

[44] Q. Xia, T. Shi, M.Y. Wang, S. Liu, A level set based method for the optimization of cast part, Structural and Multidisciplinary Optimization, 41(5), 735-747 (2010).

[45] P. Zhang, J. Liu, A. To, Role of anisotropic properties on topology optimization of additive manufactured load bearing structures, Scripta Materialia, 135, 148-152 (2017). 